\title{
The lipogenic transcription factor ChREBP dissociates hepatic steatosis from insulin resistance in mice and humans
}

\author{
Fadila Benhamed,1,2,3 Pierre-Damien Denechaud,1,2,3 Maud Lemoine,4,5,6 Céline Robichon,1,2,3 \\ Marthe Moldes,1,2,3 Justine Bertrand-Michel,7 Vlad Ratziu, 6 Lawrence Serfaty, ,,5,6 \\ Chantal Housset,4,5 Jacqueline Capeau,4,5 Jean Girard,1,2,3 Hervé Guillou, ${ }^{8}$ and Catherine Postic1,2,3 \\ ${ }^{1}$ INSERM, U1016, Institut Cochin, Paris, France. ${ }^{2}$ CNRS, UMR8104, Paris, France. ${ }^{3}$ Université Paris Descartes, Sorbonne Paris Cité, \\ Paris, France. ${ }^{4}$ Université Pierre et Marie Curie - Paris 6, UMR-S938, Paris, France. 5 Inserm U938, Centre de Recherche Saint-Antoine, \\ Paris, France. ${ }^{6}$ Assistance Publique-Hôpitaux de Paris, Hôpital Saint-Antoine, Service d’Hépatologie, Paris, France. \\ 7Plateau de lipidomique, Bio-Medical Federative Research Institute of Toulouse, INSERM, Plateforme MetaToul, \\ Toulouse, France. ${ }^{8}$ INRA-ToxAlim, Toxicologie Intégrative et Métabolisme, Toulouse, France.
}

\begin{abstract}
Nonalcoholic fatty liver disease (NAFLD) is associated with all features of the metabolic syndrome. Although deposition of excess triglycerides within liver cells, a hallmark of NAFLD, is associated with a loss of insulin sensitivity, it is not clear which cellular abnormality arises first. We have explored this in mice overexpressing carbohydrate responsive element-binding protein (ChREBP). On a standard diet, mice overexpressing ChREBP remained insulin sensitive, despite increased expression of genes involved in lipogenesis/fatty acid esterification and resultant hepatic steatosis (simple fatty liver). Lipidomic analysis revealed that the steatosis was associated with increased accumulation of monounsaturated fatty acids (MUFAs). In primary cultures of mouse hepatocytes, ChREBP overexpression induced expression of stearoyl-CoA desaturase 1 (Scd1), the enzyme responsible for the conversion of saturated fatty acids (SFAs) into MUFAs. SFA impairment of insulin-responsive Akt phosphorylation was therefore rescued by the elevation of Scd1 levels upon ChREBP overexpression, whereas pharmacological or shRNA-mediated reduction of Scd1 activity decreased the beneficial effect of ChREBP on Akt phosphorylation. Importantly, ChREBP-overexpressing mice fed a high-fat diet showed normal insulin levels and improved insulin signaling and glucose tolerance compared with controls, despite having greater hepatic steatosis. Finally, ChREBP expression in liver biopsies from patients with nonalcoholic steatohepatitis was increased when steatosis was greater than $50 \%$ and decreased in the presence of severe insulin resistance. Together, these results demonstrate that increased ChREBP can dissociate hepatic steatosis from insulin resistance, with beneficial effects on both glucose and lipid metabolism.
\end{abstract}

\section{Introduction}

Nonalcoholic fatty liver disease (NAFLD) is gaining increasing recognition as a component of the epidemic of obesity worldwide. NAFLD is the most common cause of liver dysfunction and affects close to 20 million of patients in the USA (1). The spectrum of NAFLD ranges from simple fatty liver (hepatic steatosis), with benign prognosis, to a potentially progressive form, nonalcoholic steatohepatitis (NASH), which may lead to liver fibrosis and cirrhosis, resulting in increased morbidity and mortality. All features of the metabolic syndrome, including obesity, type 2 diabetes, arterial hypertension, and hyperlipidemia (in the form of elevated triglyceride [TG] levels), are associated with NAFLD/NASH (2). Despite being potentially severe, little is known about the natural history or prognostic significance of NAFLD.

Excessive accumulation of TGs in hepatocytes is the hallmark of NAFLD $(3,4)$. Despite the existing correlation between fatty liver and insulin resistance, it remains unclear whether insulin resistance causes the excessive accumulation of TGs in liver or whether the increase in TG itself or of metabolic intermediates

Authorship note: Fadila Benhamed and Pierre-Damien Denechaud contributed equally to this work.

Conflict of interest: The authors have declared that no conflict of interest exists. Citation for this article: J Clin Invest. 2012;122(6):2176-2194. doi:10.1172/JCI41636 may play a causal role in the development of hepatic or systemic insulin resistance. Studies have favored the concept that the accumulation of intra-hepatic lipids precedes the state of insulin resistance, while others have shown that hepatic TGs themselves are not toxic and may in fact protect the liver from lipotoxicity by buffering the accumulation of deleterious fatty acids (5-8). Such findings suggest that hepatic steatosis is not necessarily associated with insulin resistance (9-11). In agreement with this concept, an identified population of obese humans can stay free of insulin resistance and are metabolically healthy despite morbid obesity (12). These findings suggest that not all lipids are detrimental for insulin sensitivity and that specific lipid species, when present in the proper location and time, may trigger signals that modulate adaptation to stress $(13,14)$.

The sources of fat contributing to fatty liver include peripheral TGs stored in white adipose tissue that flow to the liver by way of plasma nonesterified fatty acids (NEFAs), dietary fatty acids, and fatty acids produced de novo through hepatic lipogenesis. After the esterification step, TGs can either be stored as lipid droplets within hepatocytes or secreted into the blood as VLDL, but, depending on the nutritional status, they can also be hydrolyzed to allow the channeling of fatty acids toward the $\beta$-oxidation pathway (15). Using a multiple-stable-isotope approach, Donnelly et al. (16) estimated that, of the TG content accounted for 
A

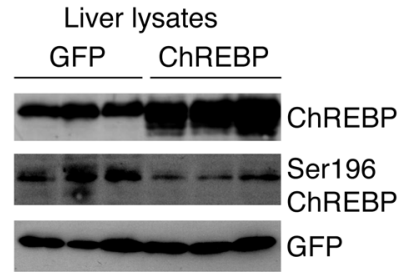

D

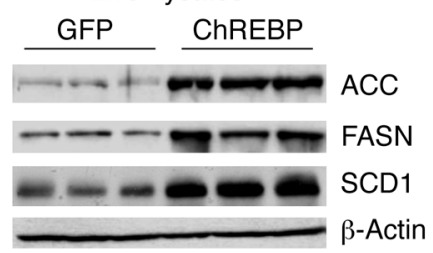

B

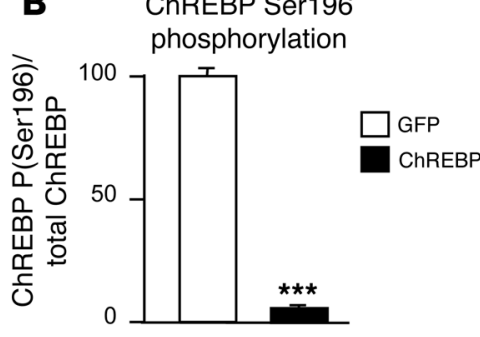

C Nuclear liver extracts

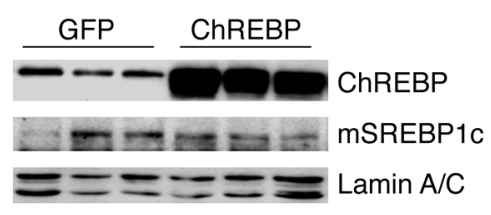

E Glycolysis, lipogenesis, and TG synthesis mRNA
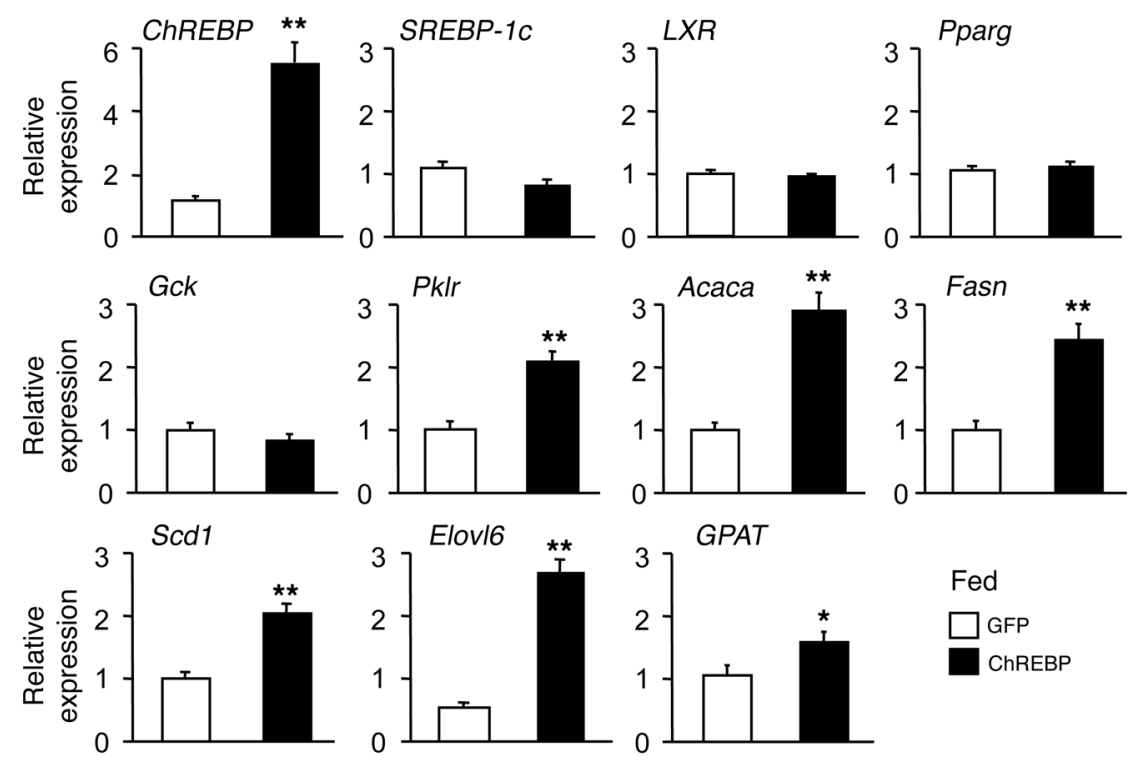

Fed

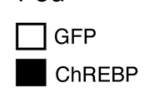

Figure 1

Overexpression of ChREBP in livers of mice leads to the induction of the entire lipogenic and esterification program. C57BL/6J mice were injected intravenously with a single dose of $5 \times 10^{9}$ pfu of GFP or ChREBP adenovirus at day 1. Four weeks later, mice were sacrificed and analyses were performed. (A) Ser196 phosphorylation level of ChREBP in livers of overnight fasted GFP and ChREBP mice. A representative Western blot is shown ( $n=10-12$ group). (B) Quantification of the ratio of Ser196 ChREBP phosphorylation compared with total ChREBP protein content is shown. ${ }^{* \star} P<0.001$ ChREBP versus GFP mice. (C) Nuclear ChREBP and SREBP-1c protein content in nuclear extracts from fed GFP versus ChREBP mice. Lamin A/C antibody was used as a loading control. A representative Western blot is shown $(n=10-12 / g r o u p)$. mSREBP-1c, mature SREBP-1C. (D) Total ACACA, FASN, and SCD1 protein content in liver lysates from fed GFP and ChREBP mice. $\beta$-Actin antibody was used as a loading control. A representative Western blot is shown ( $n=10-12 /$ group). (E) qRT-PCR analysis of ChREBP, SREBP-1c, $L X R$, Pparg, Gck, Pklr, Acaca, Fasn, Scd1, Elovl6, and GPAT in livers of GFP versus ChREBP mice. Results are the mean \pm SEM ( $n=10-12 / g r o u p)$. ${ }^{\star} P<0.05,{ }^{* \star} P<0.01$ ChREBP versus GFP mice.

in the livers of patients with NAFLD, $60 \%$ arose from NEFA, a little over $10 \%$ came from the diet, and close to $30 \%$ came from de novo lipogenesis, underlying the importance of de novo TG synthesis in the etiology of NAFLD. Pharmacologic or genetic manipulation of key lipogenic enzymes in mice can have profound metabolic consequences (reviewed in ref. 15). However, whether endogenously synthesized lipids per se affect insulin sensitivity is not clear.

Over recent years, studies reported that the liver transcription factor carbohydrate responsive element-binding protein (ChREBP) is required for the induction of the glycolytic enzyme L-pyruvate kinase (L-PK) by glucose and that acting in synergy with sterol regulatory element-binding protein-1c (SREBP-1c) stimulates lipogenic genes (acetyl-CoA carboxylase [Acaca] and fatty acid synthase $[F A S N]$ ) in response to insulin and glucose, respectively (17-19). The activation of ChREBP in response to glucose involves several posttranslational modifications, such as O-GlcNAcylation (20), acetylation on specific lysine (Lys672) (21), and dephosphorylation on serine (Ser) and threonine (Thr) residues (22). We have recently reported that modulating Ser196 phosphorylation is important for ChREBP intracellular localization and activity in response to glucose and/or glucagon $(23,24)$. The role of ChREBP in the control of glucose and lipid homeostasis is rather complex, since, on one hand, global ChREBP deficiency leads to impaired glucose tolerance and insulin resistance in C57BL/6J mice (25), and, on the other hand, ChREBP deficiency in an obese $o b / o b$ background improves hepatic steatosis and other related metabolic alterations, including insulin resistance $(26,27)$. These opposite phenotypes may reside in the fact that ChREBP controls both glycolysis and lipogenesis and that the beneficial effect of ChREBP deficiency may only be apparent in the context of lipid overload. Given these apparent contradictory effects, we thought that a better knowledge of the role of ChREBP in the control of hepatic metabolism was needed. 
A

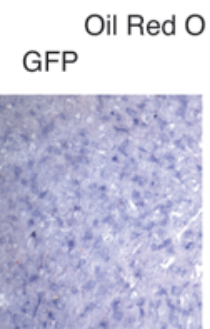

C

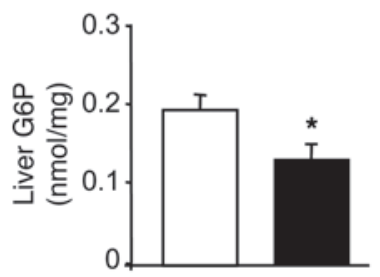

E

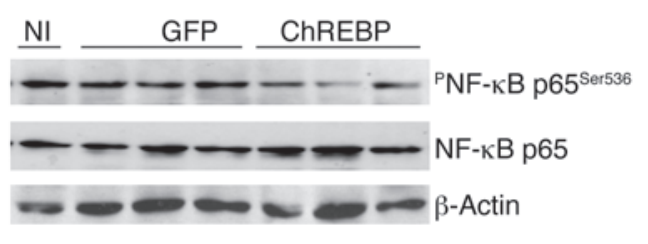

F
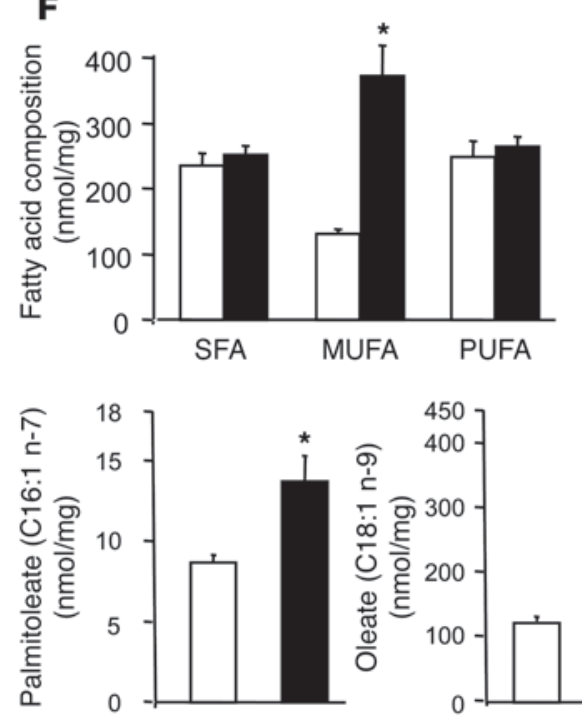

ChREBP
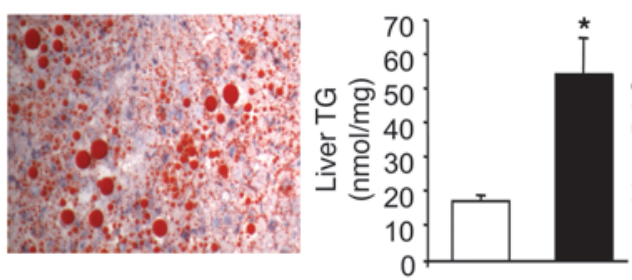

D

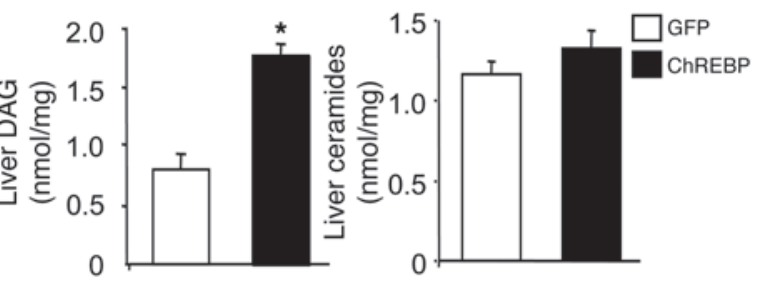

ChREBP

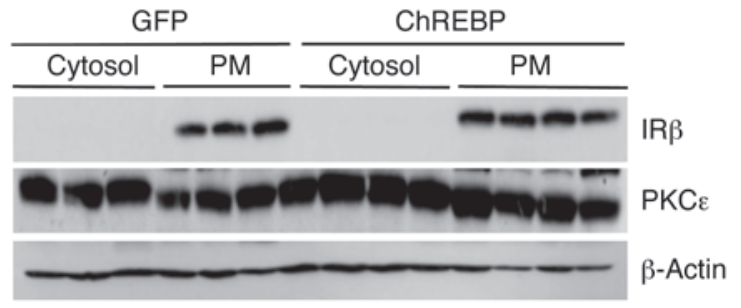

NF-kB p65 phosphorylation

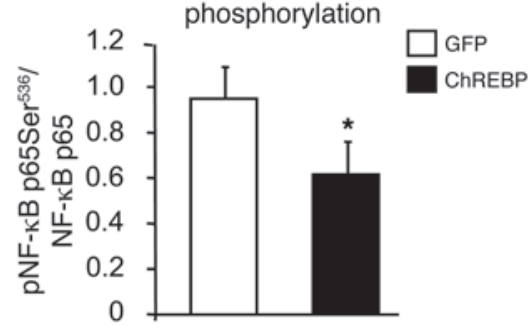

G
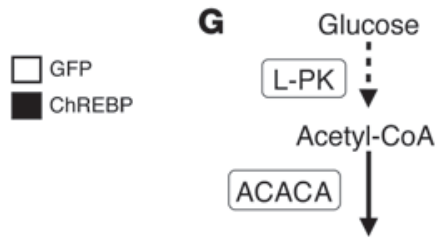

Malonyl-CoA

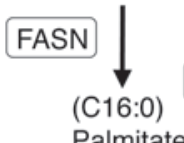

$\stackrel{\mathrm{SCD} 1}{\longrightarrow}$

Palmitate

C16:1 n-7)

Palmitoleate

Elovi6

ChREBP

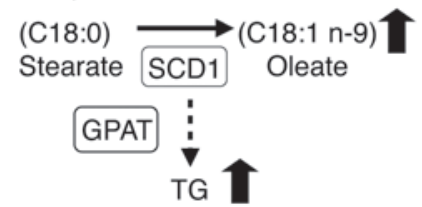

Figure 2

Overexpression of ChREBP leads to modification in hepatic lipid composition. All analyses were carried out in fed GFP and ChREBP mice. Results are the mean \pm SEM $(n=10-12 /$ group, unless specified). (A) Oil Red O staining of liver sections (original magnification, $\times 40)$. (B) Liver TGs, DAG, and ceramide concentrations. (C) Liver G6P and glycogen content. (D) PKC $\varepsilon$ content in cytosol and plasma membrane (PM) from GFP and ChREBP mice. Insulin receptor antibody (IR $\beta$ ) was used as a control of plasma membrane preparation purity, and $\beta$-actin antibody was used as a loading control. A representative Western blot is shown ( $n=6-10 / g r o u p)$. (E) Phosphorylation levels of NF-кB on Ser536 and total NF-кB p65 protein concentrations in livers of noninjected (NI), GFP, and ChREBP mice. A representative Western blot is shown ( $n=6 / g r o u p)$. Quantification of NF-кB p65 Ser536/NF-кB p65 is shown ( $n=6$ /group). (F) Fatty acid composition and palmitoleate (C16:1 $n-7)$ and oleate $(C 18: 1 n-9)$ concentrations. PUFAs, polyunsaturated fatty acids. (G) Schematic representation of the enzymatic steps involved in fatty acid synthesis from glucose in liver. The enzymes controlled by ChREBP are indicated: L-PK, ACACA, FASN, SCD1, Elovl6, and GPAT. A black arrow shows the change in fatty acid composition caused by ChREBP overexpression. ${ }^{*} P<0.05$ ChREBP versus GFP. 
Table 1

Metabolic parameters in GFP and ChREBP mice under standard chow and HFDs

\begin{tabular}{lcccc} 
& \multicolumn{3}{c}{ CD } & HFD \\
Mice & GFP & ChREBP & GFP & ChREBP \\
Body weight (g) & $23.78 \pm 0.54$ & $24.56 \pm 1.16$ & $30.9 \pm 2.3$ & $29.7 \pm 1.4$ \\
Liver weight (g) & $1.40 \pm 0.07$ & $1.83 \pm 0.07 \mathrm{~A}$ & $1.45 \pm 0.40$ & $2.41 \pm 0.12^{\mathrm{A}}$ \\
White adipose weight (epididymal) (g) & $0.38 \pm 0.03$ & $0.16 \pm 0.02^{\mathrm{A}}$ & $0.65 \pm 0.05$ & $0.46 \pm 0.05^{\mathrm{A}}$ \\
Glucose (fed [mM]) & $8.30 \pm 0.29$ & $7.22 \pm 0.39$ & $10.8 \pm 1.5$ & $9.9 \pm 0.8$ \\
Glucose (fasting [mM]) & $5.19 \pm 0.21$ & $4.52 \pm 0.25^{\mathrm{A}}$ & $6.95 \pm 0.19$ & $4.95 \pm 0.22^{\mathrm{A}}$ \\
Serum insulin (fasting [ng/ml]) & $0.39 \pm 0.10$ & $0.27 \pm 0.04 \mathrm{~A}$ & $7.61 \pm 3.20$ & $0.77 \pm 0.54^{\mathrm{A}}$ \\
Serum TGs (mmol/l) & $0.56 \pm 0.20$ & $0.54 \pm 0.46$ & $0.76 \pm 0.06$ & $0.84 \pm 0.08$ \\
NEFAs (mg/dl) & $0.79 \pm 0.26$ & $0.68 \pm 0.05$ & $1.66 \pm 0.90$ & $0.88 \pm 0.08^{\mathrm{A}}$ \\
$\beta$ Hydroxybutyrate (nM) & $0.22 \pm 0.03$ & $0.18 \pm 0.04$ & $0.32 \pm 0.02$ & $0.24 \pm 0.03^{\mathrm{A}}$ \\
AST (U/l) & $280 \pm 18$ & $149 \pm 12^{\mathrm{A}}$ & $286 \pm 33$ & $283 \pm 21$ \\
ALT (U/l) & $598 \pm 20$ & $526 \pm 18$ & $999 \pm 23$ & $803 \pm 27$ \\
\hline All measurements were carried out with 14-week-old mice $(n>6)$. All data are presented as mean $\pm \mathrm{SEM} .{ }^{\mathrm{A} P}<0.05$.
\end{tabular}

(Elovl6) as well as esterification (GPAT) was significantly increased in livers of ChREBP mice compared with those of GFP controls (Figure 1E). The induction of Acaca, Fasn, and $S c d 1$ gene expression led to a parallel increase in total protein content (Figure 1D). Overexpression of ChREBP also led to the induction of glycolytic and lipogenic genes (Pklr, Fasn, Acaca, Scd1) under fasting conditions (Supplemental Figure 2A).

The observed changes in gene expression occurred without influencing liver $X$ receptor $\alpha(L X R)$ or Pparg expres-

In this study, we determined the metabolic impact of enhancing liver lipogenic rates via ChREBP on the outcome of hepatic steatosis and/or insulin resistance. We showed that while stimulating the entire lipogenic/esterification program, the adenoviral-mediated overexpression of ChREBP in mouse liver does not lead to insulin resistance. The dissociation between ChREBP-mediated steatosis and hepatic insulin resistance was even more apparent under high-fat diet (HFD) feeding. Lipidomic analysis revealed that ChREBP-mediated hepatic steatosis was not deleterious and that beneficial lipid species (monounsaturated fatty acids [MUFAs]) were preferentially enriched. Lastly, we measured the expression of ChREBP in livers of a small cohort of patients with histologically proven NASH. We compared the hepatic levels of CHREBP mRNA, according to the degree of steatosis and the severity of insulin resistance. Here, we also report a significant correlation among hepatic ChREBP expression, liver steatosis, and insulin resistance in humans.

\section{Results}

Overexpression of ChREBP in liver leads to the induction of the entire lipogenic and esterification program. To gain insights into the role of ChREBP in lipogenesis and insulin resistance, an adenovirus that expresses a dephosphorylated isoform of ChREBP (DP) was generated (19). Although we recently reported the importance of ChREBP acetylation and O-GlcNAcylation in response to glucose $(20,21)$, we also described the relevance of modulating the phosphorylation of Ser196 residue for ChREBP localization and/or activity in response to glucose and glucagon $(23,24)$. The phosphorylation level of the dephosphorylated ChREBP isoform was revealed using a specific ChREBP Ser196 antibody (Figure 1, A and B). Four weeks after adenoviral injection (1 single dose of $5 \times 10^{9}$ plaque-forming units/mice [pfu/mice]), high levels of GFP expression were detected in livers of both GFP- and ChREBP-injected mice but not in other tested tissues (Supplemental Figure 1A; supplemental material available online with this article; doi:10.1172/ JCI41636DS1). ChREBP total protein (Figure 1A) and nuclear content (Figure 1C) were markedly increased in livers from ChREBP mice compared with that in GFP controls but not in other sites of ChREBP expression (i.e., white adipose tissue or skeletal muscle) (Supplemental Figure 1B). As a result, expression of key genes of glycolysis (Pklr), lipogenesis (Acaca, Fasn, Scd1), elongation sion (Figure 1E). In addition, no modification in mature SREBP-1c protein content was observed (Figure 1C). Consistent with this observation, glucokinase (Gck) expression, which is primarily under the transcriptional control of insulin via SREBP-1c (28) remained unchanged under ChREBP overexpression (Figure 1E).

Overexpression of ChREBP leads to modification in liver lipid composition. We next determined the metabolic consequences of liverrestricted ChREBP overexpression (Figure 2). ChREBP overexpression led to significant accumulation of lipid droplets visible after Oil Red $\mathrm{O}$ staining on liver sections (Figure 2A) that was correlated to a 3 -fold increase in TG concentrations (Figure 2B). As a consequence, liver weight was increased by $25 \%$ in ChREBP mice compared with that in GFP mice (Table 1). In agreement with a redirection of the glucose flux toward fatty acid synthesis, we observed that glucose 6-phosphate (G6P) and glycogen concentrations were reduced in livers of ChREBP mice (Figure 2C). Interestingly, the weight of epididymal adipose tissue was reduced by over 50\% in ChREBP mice, and NEFA concentrations were lower (although the difference between GFP and ChREBP mice did not reach statistical significance) (Table 1). Liver damage was assessed by serum levels of liver aspartate aminotransferase (AST) and alanine aminotransferase (ALT), which leak out to the circulation with liver injury. Intriguingly, AST concentrations were lower in ChREBP mice (Table 1), despite enhanced lipogenic gene program and hepatic steatosis (Figure $1 \mathrm{E}$ and Figure 2B). Consistent with potential lower liver damage, the level of NF- $\kappa B$ p 65 phosphorylation, reflecting the activity of NF- $\mathrm{KB}$, was significantly lower in livers of ChREBP mice (Figure 2E).

Because fatty acids have been shown to serve as signaling molecules, exerting either beneficial (i.e., lipokines) or detrimental (i.e., lipotoxicity) metabolic outcomes $(8,29,30)$, lipidomic analysis was performed in livers of ChREBP mice. No difference in ceramide concentrations was observed between GFP and ChREBP mice (Figure 2B). Accumulation of diacylglycerol (DAG) has been reported to cause insulin resistance by activating a specific isoform of protein kinase C, $\mathrm{PKC} \varepsilon(8,31,32)$. Surprisingly, despite a 3-fold increase in DAG concentrations in livers of ChREBP mice (Figure 2B), no modification in PKCE localization in the cytosol and/or in plasma membranes of livers from ChREBP mice was observed (Figure 2D). Interestingly, lipidomics revealed a 3-fold increase in MUFA content in livers of ChREBP mice without any modification of the 


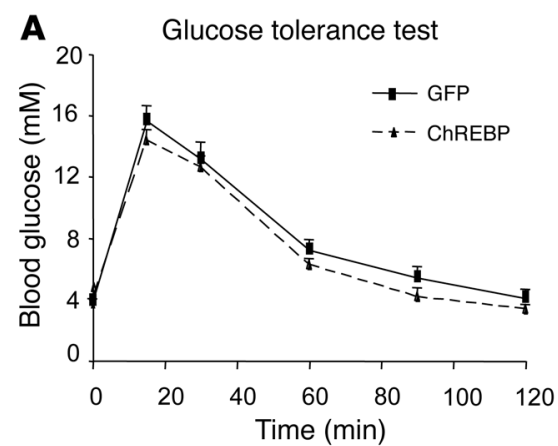

B
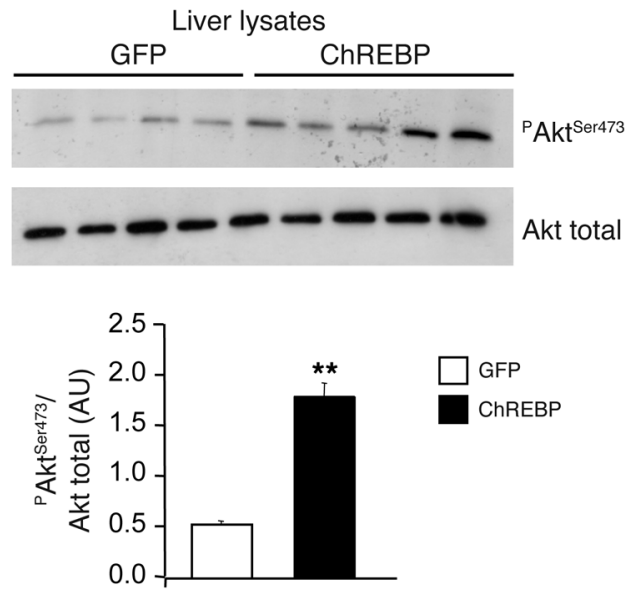

D Gluconeogenesis and $\beta$-oxidation mRNA

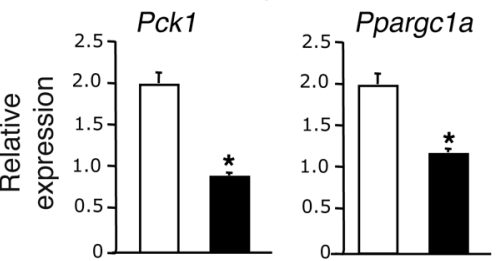

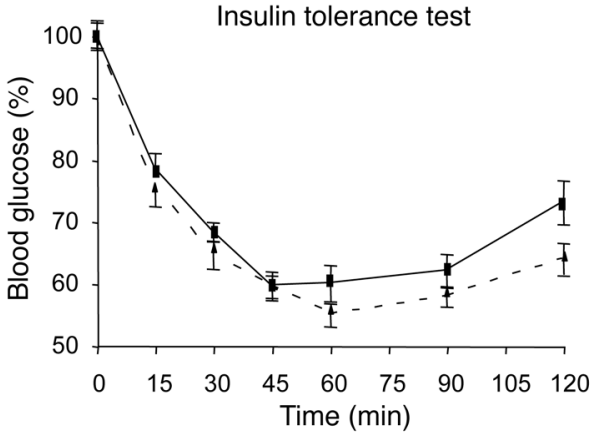

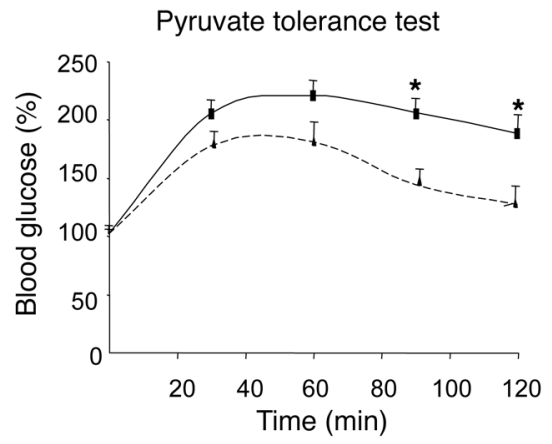

C Liver lysates
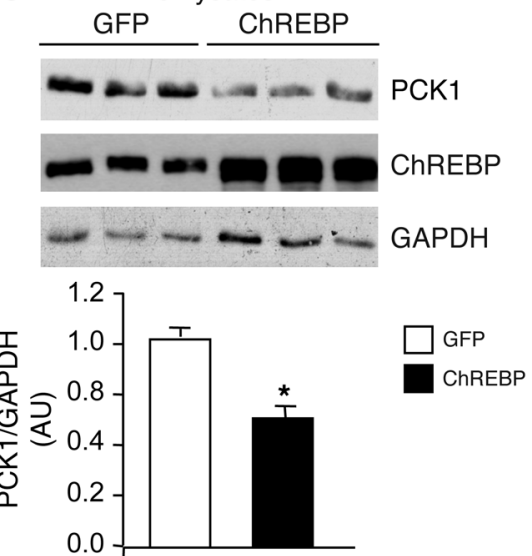

Figure 3

ChREBP-induced steatosis is not associated with insulin resistance. (A) Glucose tolerance $(1 \mathrm{~g} / \mathrm{kg})$, insulin tolerance $(0.75 \mathrm{U} / \mathrm{kg})$, and pyruvate tolerance $\left(2 \mathrm{~g} / \mathrm{kg}\right.$ ) tests were performed in GFP and ChREBP mice. ${ }^{*} P<0.05$ ChREBP versus GFP mice. (B) Representative Western blot analysis of Ser473 phosphorylation in liver of fasted GFP and ChREBP mice. Quantification of the ratio of Ser473 Akt phosphorylation compared with total Akt protein content is shown. ${ }^{* *} P<0.01$ ChREBP versus GFP mice ( $n=6-10$ mice/group). (C) Total PCK1 and ChREBP protein content in liver lysates from overnight fasted GFP and ChREBP mice. GAPDH antibody was used as a loading control. A representative Western blot is shown ( $n=6-8 /$ group). Quantification of the ratio of PCK1 compared with GAPDH content is shown. ${ }^{*}<0.05$ ChREBP versus GFP mice ( $n=6-10$ mice/group). (D) qRT-PCR analysis of Pck1, Ppargc1a, Foxo1, Cpt1a, Ppara, and Fgf21 in livers from overnight fasted GFP and ChREBP mice. Results are the mean \pm SEM ( $n=6-8$ mice/group). ${ }^{\star} P<0.05,{ }^{\star \star} P<0.01$ ChREBP versus GFP.

concentrations of either saturated fatty acids (SFAs) or polyunsaturated fatty acids (Figure $2 \mathrm{~F}$ ). It should be noted that a specific increase in palmitoleate (C16:1, n-7) and oleate (C18:1, n-9) concentrations was observed (Figure $2 \mathrm{~F}$ ), in agreement with the significant effect of ChREBP overexpression on SCD1 expression (Figure 1D and Figure 2G).

ChREBP-induced steatosis is not associated with insulin resistance. To determine whether the development of hepatic steatosis affected whole glucose homeostasis in ChREBP mice in vivo, glucose, insulin, and pyruvate tolerance tests were performed (Figure $3 \mathrm{~A}$ ). While no statistical difference was reached for glucose or insulin tolerance between GFP and ChREBP mice, a difference was observed for pyruvate tolerance (Figure $3 \mathrm{~A}$ ). While plasma glu- cose rose significantly for both GFP and ChREBP mice after the injection of pyruvate, pyruvate conversion into glucose was lower in ChREBP mice compared with that in GFP mice throughout the time course of the test (Figure 3A). This difference, which reflects lower rates of gluconeogenesis, was in agreement with lower fasting blood glucose concentrations measured in ChREBP mice (Table 1) and with the significant decrease in expression of hepatic PCK1, the rate-limiting enzyme of gluconeogenesis whose expression is inhibited by insulin (Figure 3, C and D). The expression of Ppargc1a, which coactivates a number of transcription factors, such as hepatic nuclear factor- $4 \alpha$ and forkhead box O1 (Foxo1), was also significantly decreased in livers of ChREBP mice. Foxo1 mRNA levels were also decreased by 50\% (Figure 3D). 
A

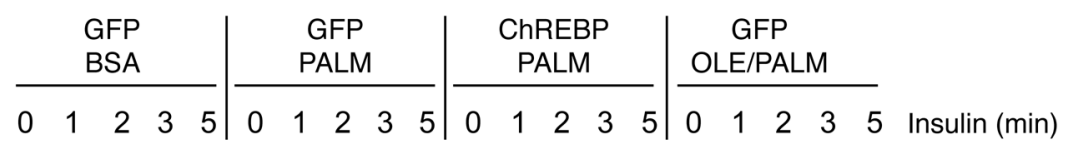
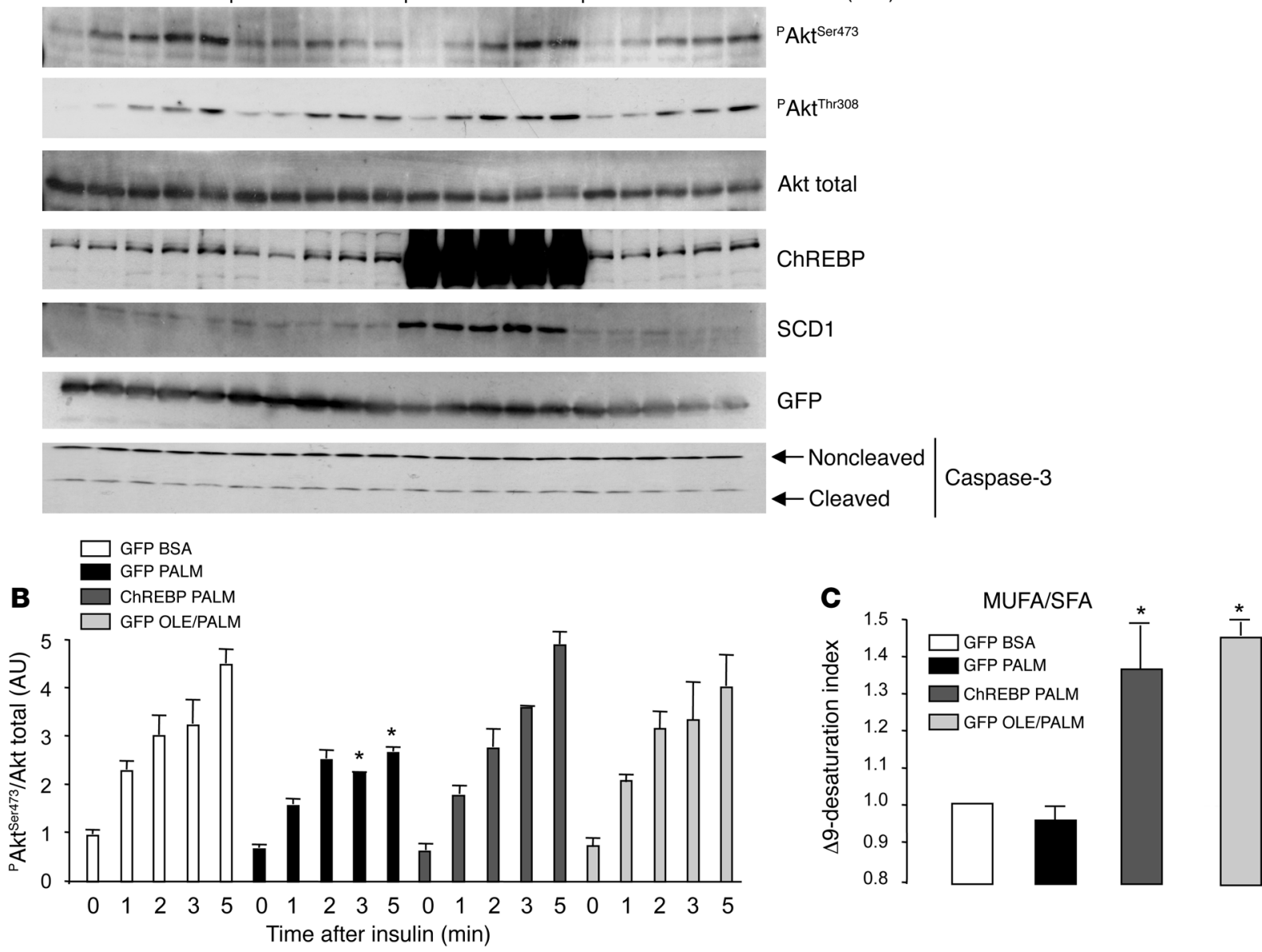

\section{Figure 4}

ChREBP overexpression protects against PALM-induced insulin resistance in primary cultured hepatocytes. Twenty-four hours after plating, mouse hepatocytes were infected with 3 pfu of GFP or ChREBP adenovirus before being incubated for 24 hours with 0.48 mM albumin-bound PALM or $0.48 \mathrm{mM}$ albumin-bound OLE/PALM, as described in the Methods section. An insulin (1 $\mathrm{nM}$ ) time course was performed for 1, 2, 3, or 5 minutes. (A) Western blot analysis of insulin-mediated Akt phosphorylation on Ser473 and Thr308. Total Akt, ChREBP, SCD1, GFP, noncleaved caspase-3, and cleaved caspase-3 protein content was determined under similar culture conditions. Representative Western blots are shown ( $n=6-10$ independent cultures). (B). Quantification of the ratio of Ser473 Akt phosphorylation compared with total Akt protein content is shown. ${ }^{*} P<0.05$ GFP PALM versus GFP BSA mice for equivalent time point ( $n=6-10$ independent cultures). (C). The $\Delta 9$-desaturation index was calculated as described in the Methods section. ${ }^{*} P<0.05$ versus GFP PALM.

Modification in liver gene expression was correlated with a 3-fold increase in protein kinase B/Akt Ser 473 phosphorylation under fasting conditions (Figure 3B). This increase in Akt Ser473 phosphorylation occurred independently of an increase in insulin concentrations in fasted ChREBP mice, suggesting enhanced insulin sensitivity in these mice (Table 1). In contrast, when a supraphysiological dose of insulin was injected into GFP and ChREBP mice, no apparent modification of the insulin-mediated phosphorylation of Akt (Ser473) or of GSK3 $\beta$ (Ser9) was observed in livers of ChREBP mice compared with that in controls (Supplemental Figure 2B).

Since conditions associated with high rates of lipogenesis are associated with a shift in cellular metabolism from fatty acid $\beta$-oxidation to TG esterification, we examined mRNA levels of Cpt1a, the rate-limiting enzyme of mitochondrial $\beta$-oxidation, and
Ppara, a nuclear receptor that promotes $\beta$-oxidation. The expression of Cpt1a and Ppara was significantly decreased in livers of fasted ChREBP mice compared with that of GFP controls, suggesting decreased mitochondrial $\beta$-oxidation rates in these mice (Figure 3D). We also measured the expression of fibroblast growth factor 21 (FGF21), a key metabolic regulator whose expression is regulated via PPAR pathways during states requiring increased fatty acid oxidation (33). Fgf21 mRNA levels were 3-fold increased in livers of ChREBP mice, despite decreased Ppara gene expression (Figure 3D). Our results suggest that the transcriptional control exerted by ChREBP on FGF21 (34) may be dominant over that of PPAR and that increased FGF21 expression did not lead to increased mitochondrial $\beta$-oxidation rates under our experimental conditions. Altogether, our results suggest that ChREBP-mediated hepatic steatosis is dissociated from insulin resistance in mice. 


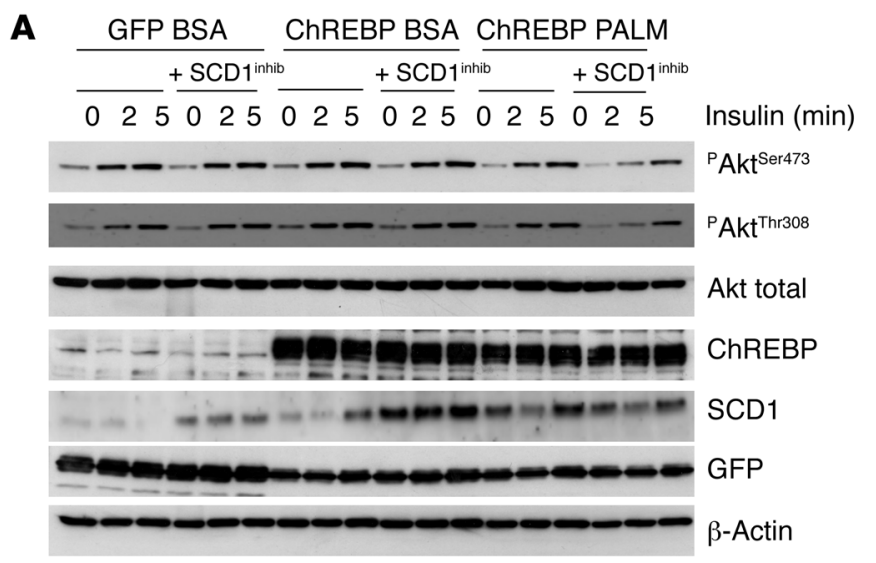

B

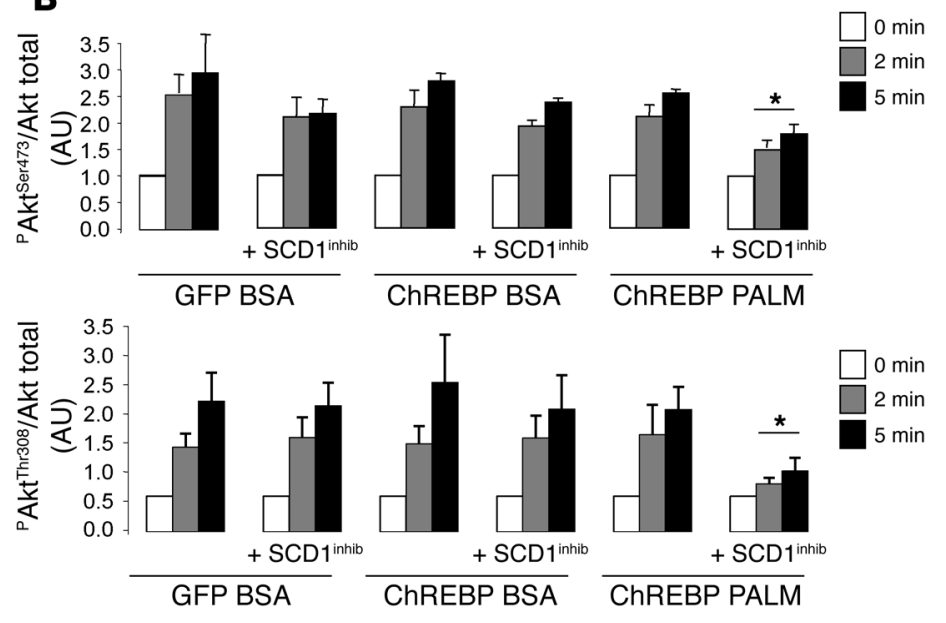

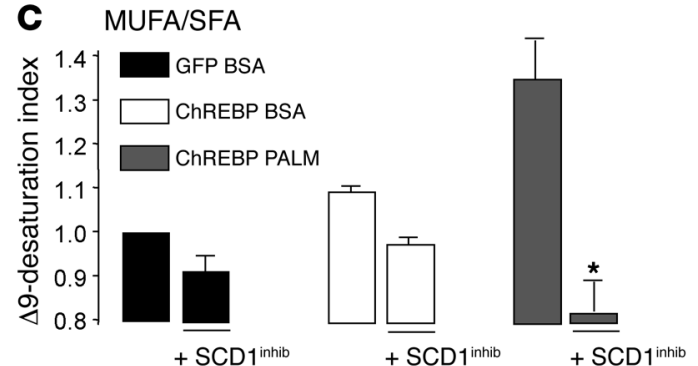

\section{Figure 5}

Inhibition of SCD1 activity in hepatocytes attenuates the protective effect of ChREBP on Akt phosphorylation. Twenty-fours hour after plating, mouse hepatocytes were infected with 3 pfu of GFP or ChREBP adenovirus before being incubated for 24 hours with $0.48 \mathrm{mM}$ albumin-bound PALM, with or without $10 \mathrm{nM} \mathrm{SCD1}$ inhibitor (SCD1 inhib) (36), as indicated. An insulin (1 nM) time course was performed for 2 and 5 minutes. (A) Western blot analysis of insulin-mediated Akt phosphorylation on Ser473, Thr308, total Akt, ChREBP, SCD1, and GFP protein content. Representative Western blots are shown ( $n=4$ independent cultures). $\beta$-Actin was used as a loading control. (B) Quantification of the ratio of Ser473 and Thr308 Akt phosphorylation compared with total Akt protein content is shown. ${ }^{*} P<0.05$ ChREBP PALM plus SCD1inhib versus ChREBP PALM. (C) The $\triangle 9$-desaturation index was measured under similar culture conditions as indicated. ${ }^{*} P<0.05$ ChREBP PALM plus SCD1 $1^{\text {inhib }}$ versus ChREBP PALM.

ChREBP overexpression protects against palmitate-induced insulin resistance in primary cultured hepatocytes. Next, we determined whether the increase in hepatic MUFA concentrations measured in livers of ChREBP mice may account for the lack of lipotoxicity and insulin resistance. To address this issue, we tested whether a selective ChREBP-mediated increase in MUFA concentrations (such as oleate) could protect against SFA-mediated insulin resistance in primary hepatocytes. Mouse hepatocytes were first infected with either 3 pfu/cell of GFP or ChREBP adenovirus for 24 hours and then incubated in the presence of $0.48 \mathrm{mM}$ palmitate (PALM, C16:0) for another 24 hours. Hepatocytes infected with GFP ( $3 \mathrm{pfu} / \mathrm{cell})$ and incubated with a mix of oleate/PALM (OLE/PALM) for 24 hours were used as controls (Figure 4). After adenoviral treatment, insulin $(1 \mathrm{nM})$ was added for times ranging from 0 to 5 minutes. The insulin-mediated phosphorylation of Akt on Ser473 was significantly reduced in the presence of PALM (at time points 3 and 5 minutes) (Figure 4, A and B). The PALM-mediated decreased Akt phosphorylation was not due to cell apoptosis, since no significant increase in cleaved caspase- 3 was detected under all the tested culture conditions (Figure 4A). The weak cleaved caspase- 3 band was likely due to the adenoviral infection, since it was equivalent regardless of the culture conditions (Figure 4A). Interestingly, when ChREBP was overexpressed, the deleterious effect of PALM on insulin-mediated phosphorylation of Akt on Ser473 and on Thr308 was prevented (Figure 4A) and was associated with a marked induction of SCD1 (the $\triangle 9$-desaturase), the enzyme responsible for the conversion of SFA to MUFA (35) (Figure 4A). As a result, a significant increase in $\triangle 9$-desaturation index, reflecting the MUFA/SFA ratio, was observed under these experimental conditions (Figure 4C). In agreement with this hypothesis, a similar protective effect was observed when hepatocytes were incubated in the presence of a combination of OLE/PALM (Figure 4, B and C).

To better define the contribution of SCD1 to the ChREBP-mediated protective effects on Akt phosphorylation, we pharmacologically inhibited SCD1 activity (SCD $1^{\text {inhib) }}$, as described previously (36). First, within the same set of experiments, we confirmed both 
A

BSA
GFP
GFP SCD1 $\frac{\text { BSA }}{\text { ShCtrl }} \frac{\text { PALP }}{\text { shCtrl ShSCD1 }}$

$\begin{array}{llllllllllllllllll}0 & 2 & 5 & 0 & 2 & 5 & 0 & 2 & 5 & 0 & 2 & 5 & 0 & 2 & 5 & 0 & 2 & 5\end{array}$

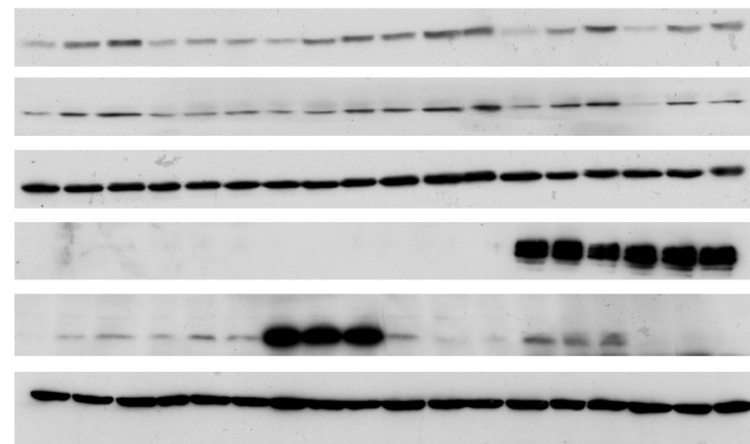

B

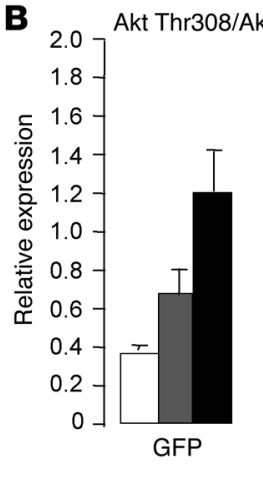

$$
\text { BSA }
$$

C

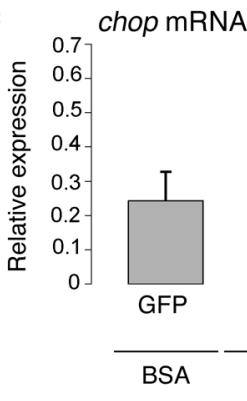

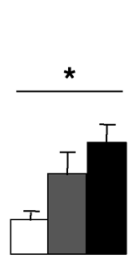

GFP

PALM

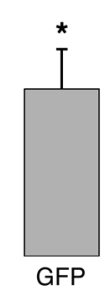

PALM

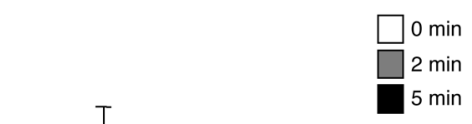

Figure 6

In vitro overexpression of SCD1 recapitulates the beneficial effect of ChREBP on Akt phosphorylation. Twenty-four hours after plating, mouse hepatocytes were infected with a combination of adenovirus as indicated (GFP, SCD1, ChREBP, shCTRL, and/or shSCD1) and described in the Methods section. Cells were then incubated for 24 hours with BSA or $0.48 \mathrm{mM}$ albumin-bound PALM. An insulin (1 $\mathrm{nM})$ time course was performed for 2 and 5 minutes ( $n=6$ independent cultures). (A) Western blot analysis of insulin-mediated Akt phosphorylation on Ser473, Thr308, total Akt, ChREBP, and SCD1. Representative Western blots are shown. $\beta$-Actin was used as a loading control. (B) Quantification of the ratio of Thr308 compared with total Akt protein content is shown. ${ }^{*} P<0.05$ compared with GFP BSA. (C) qRT-PCR analysis of chop mRNA under the indicated culture conditions. ${ }^{*} P<0.05$ GFP PALM compared with GFP BSA.

the negative effect of PALM incubation on Akt Ser473 phosphorylation (Supplemental Figure 3, A and B) as well as the protective effect of ChREBP on Akt Ser473 and Thr308 phosphorylation in the presence of PALM (Figure 5, A and B). Performed in parallel, these results are comparable but are presented in 2 separate panels. When SCD1 activity was pharmacologically inhibited in the presence of PALM
Insulin (min)

PAkt ${ }^{\text {Ser473 }}$

${ }^{\mathrm{P}} \mathrm{Akt}^{\mathrm{Th}}{ }^{\mathrm{T} 308}$

Akt total

ChREBP

SCD1

$\beta$-Actin

(Figure 5A), the protective effect of ChREBP on insulin signaling (i.e., Akt phosphorylation on Ser473 and Thr308) was significantly reduced (Figure 5B) and was correlated with a significant decrease in $\Delta 9$-desaturation index (Figure $5 \mathrm{C}$ ). The $\Delta 9$-desaturation index was only significantly affected in presence of both ChREBP and PALM (Figure 5C), the only condition also associated with significant modification of the phosphorylated Akt/Akt ratio (Figure 5B). It should be noted here that ChREBP conditions alone (i.e., without PALM) did not increase the MUFA/SFA ratio, since hepatocytes were cultured under low glucose concentrations (5 $\mathrm{mM}$ ), experimental conditions not sufficient to turn on lipogenic rates (17). Altogether, our results show that ChREBP overexpression reverses PALMinduced insulin resistance by increasing the MUFA/ SFA ratio in vitro.

To determine whether ChREBP overexpression in vitro led to metabolic changes downstream of Akt phosphorylation, basal and insulin-mediated inhibition of glucose production were measured in primary hepatocytes overexpressing ChREBP with or without the SCD1 inhibitor (Supplemental Figure 4). First, we observed that basal glucose production was increased by $20 \%$ upon PALM incubation. While insulin (100 nM) failed to decrease glucose production under PALM conditions, the presence of ChREBP enabled insulin to significantly decrease glucose production, despite the presence of PALM in the culture medium. This effect was lost when the SCD1 inhibitor was added (Supplemental Figure 4). This set of experiments confirms that ChREBP favors the effect of insulin on a key metabolic pathway such as glucose production in vitro.

Overexpression of SCD1 recapitulates the beneficial effect of ChREBP on Akt phosphorylation in vitro. AcylCoA desaturases, such as SCD1 (37), introduce a double bond at a specific position on the acyl chain of fatty acids, thereby influencing several structural, metabolic, and signaling properties of the fatty acid or of the fatty acid containing lipids. Among desaturases, SCD1 is particularly critical to convert SFAs (mainly PALM C16:0 and stearate C18:0) into MUFAs (palmitoleate C16:1n-7 and oleate C18:1n-9) in the liver (38). To better define the role of SCD1 in the beneficial effect of ChREBP on insulin signaling in liver cells, we performed experiments in which SCD1 was either overexpressed or knocked down using adenoviral strategies in primary cultured hepatocytes (Figure 6). We observed that SCD1 overexpression restored Akt phosphorylation on both Ser473 (Supplemental Figure 5) and Thr308 to control levels (Figure 6B) in the presence of PALM). In fact, SCD1 and ChREBP overexpression had similar beneficial effects on rescuing Akt phosphorylation (Figure 6, A and B) in the presence of PALM. It should be noted, however, that the levels of SCD1 reached were quite different depending on whether SCD1 or ChREBP was overexpressed (Figure 6A). Despite 
A Glycolysis, lipogenesis, and TG synthesis
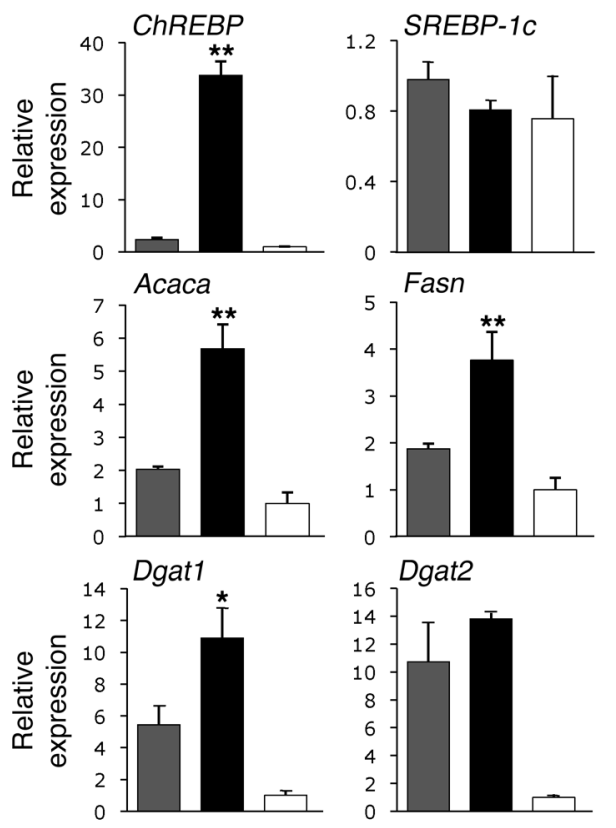

B Fat uptake, $\beta$ oxidation, and export
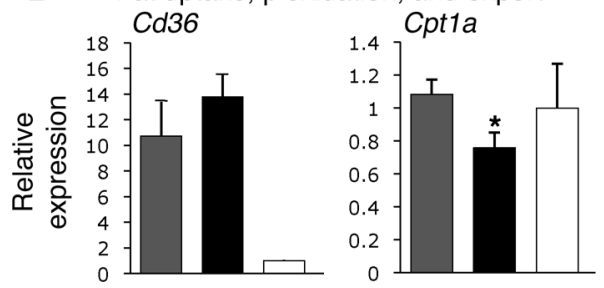

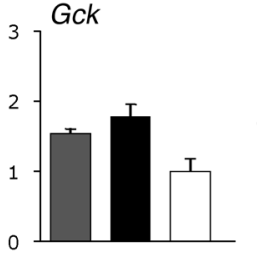

Scd1

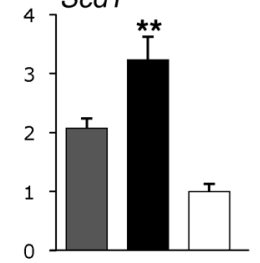

GPAT
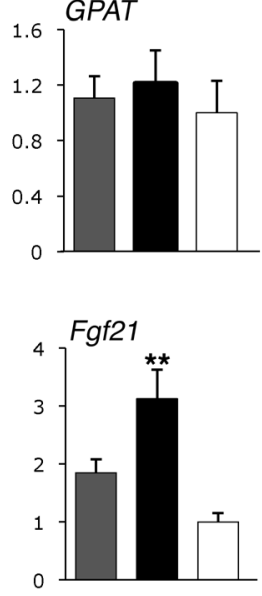

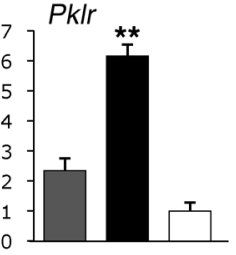

Elovi6

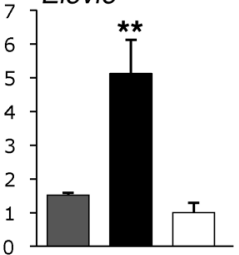

$\square$ HFD GFP

HFD ChREBP $\square$ CD GFP

\section{Figure 7}

Gene expression in livers of HFD-fed ChREBP mice. qRT-PCR analyses were carried out in HFD-fed (HFD) GFP and ChREBP mice, as described in the Methods section. GFP mice maintained on a standard CD for a similar number of weeks (10 weeks) were used as controls. Mice were sacrificed in the fasted state. (A) qRT-PCR analysis of ChREBP and genes involved in the glycolytic (Gck, Pklr), lipogenic (SREBP-1c, Acaca, Fasn, Scd1, Elovl6), and TG synthesis (Dgat1, Dgat2, GPAT) pathways. (B) qRT-PCR analysis of genes involved in fat uptake (Cd36), $\beta$-oxidation (Cpt1a, Fgf21), and export $(M T P)$. Results are the mean \pm SEM ( $n=8-10 /$ group). ${ }^{\star} P<0.05,{ }^{* \star} P<0.01$ HFD-fed ChREBP versus HFD-fed GFP mice. this difference, ChREBP efficiently rescued Akt phosphorylation to levels similar to those of SCD1 and control conditions (Figure 6, $A$ and B). Importantly, we observed that the beneficial effect of ChREBP was significantly attenuated when SCD1 was knocked down (Figure 6, A and B). Recent studies have revealed that chronic SFA exposure activates unfolded protein response (UPR) in cultured cells $(39,40)$. UPR induces transcription of a set of genes whose protein products increase the capacity for protein folding and ER-associated degradation and induces apoptosis when the ER function is severely impaired $(41,42)$. Of these genes, chop is often used as an UPR marker. As previously described (40), chop expression was increased in cells incubated with PALM (Figure 6C). Interestingly, the PALM-mediated induction of chop was prevented when SCD1 or ChREBP was overexpressed (Figure 6B). These results suggest that ChREBP and/or SCD1 prevented chop induction by increasing the MUFA/SFA ratio (as shown in Figure 5). Surprisingly, no significant reinduction of chop was observed when SCD1 was knocked down in the cells overexpressing ChREBP and incubated with PALM (Figure 6C), suggesting that ChREBP alone was sufficient to overcome the effect of PALM on chop expression.

Altogether, these experiments confirm that SCD1 overexpression can recapitulate the protective effect of ChREBP on Akt phosphorylation but also suggest that ChREBP overexpression may also exert its beneficial effects through additional mechanisms.

Exacerbated hepatic steatosis in HFD-fed ChREBP mice. Having determined that ChREBP-mediated liver steatosis was dissociated from insulin resistance under standard chow diet (CD) con-

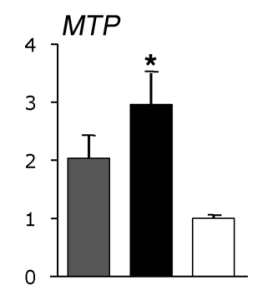

ditions, we wished to address whether ChREBP overexpression could reverse some of the metabolic alterations induced by HFD feeding. To address this issue, C57BL/6J mice were maintained on a HFD ( $60 \%$ calories coming from fat) for 10 weeks. Six weeks after the beginning of the diet, mice were injected with 1 single dose of $5 \times 10^{9}$ pfu of GFP or ChREBP adenovirus and maintained on HFD for another 4 weeks. Mice injected with a similar dose of GFP and maintained for 10 weeks on standard CD were used as controls. ChREBP overexpression markedly induced glycolytic (Pklr) and lipogenic gene expression (Acaca, Fasn, Scd1, Elovl6) in livers of HFD-fed ChREBP mice (Figure 7A). No difference in the expression of $C d 36$, involved in fatty acid uptake, was observed in livers of HFD-fed ChREBP mice compared with that in HFD-fed GFP mice (Figure 7B). In contrast, a significant decrease in Cpt1a gene expression (Figure 7B) and in $\beta$-hydroxybutyrate concentrations (Table 1) was also observed, likely reflecting a decrease in $\beta$-oxidation rates in HFD-fed ChREBP mice. As a consequence, lipid droplet accumulation, as revealed by Oil red $\mathrm{O}$ staining on liver sections (Figure 8A), and hepatic TG concentrations were further increased (a 2.5-fold increase) (Figure 8C) in HFD-fed ChREBP mice compared with those in HFD-fed GFP mice. Interestingly, while their liver weight was increased $(+40 \%)$, a marked decrease in white adipose tissue mass $(-60 \%)$ (epididymal fat depot) was observed for HFD-fed ChREBP mice (Figure 8B), a reduction that was correlated with decreased NEFA concentrations $(-40 \%)$ (Table 1). Despite exacerbated steatosis, no further increase in the expression of Tnfa, chop (Figure 8D), and of known 
A

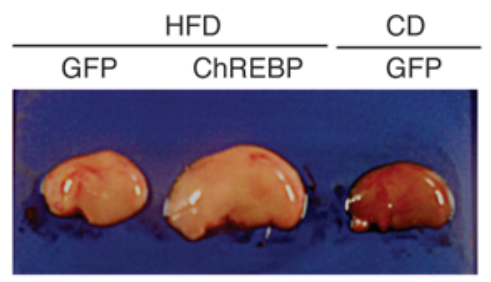

B
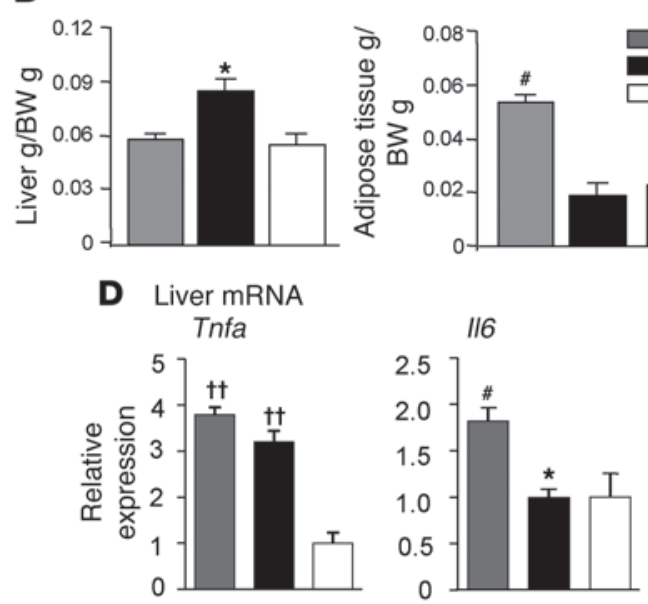

$\mathbf{F}_{60}$ Liver fatty acid composition

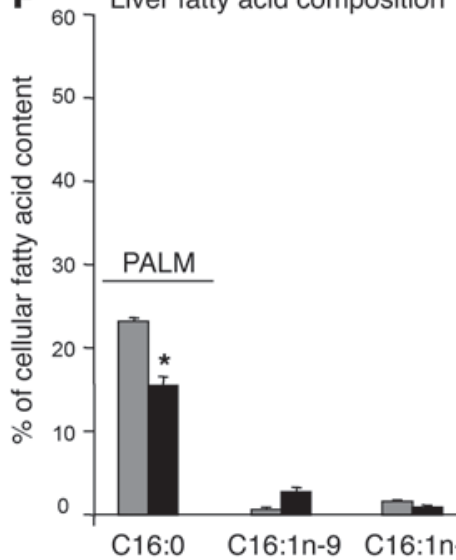

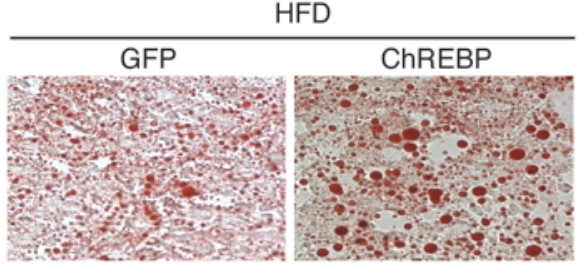

C
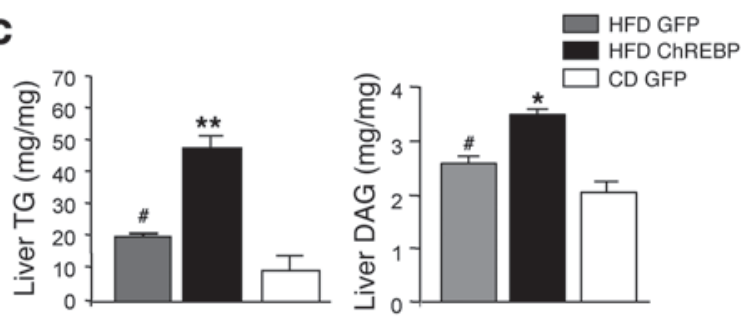

$\mathrm{CD}$

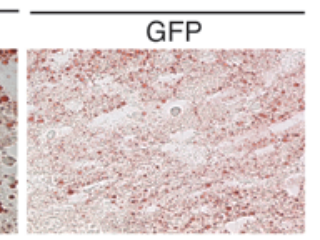

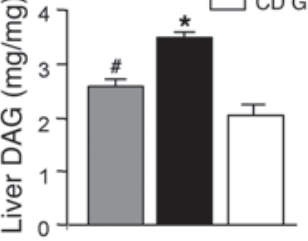
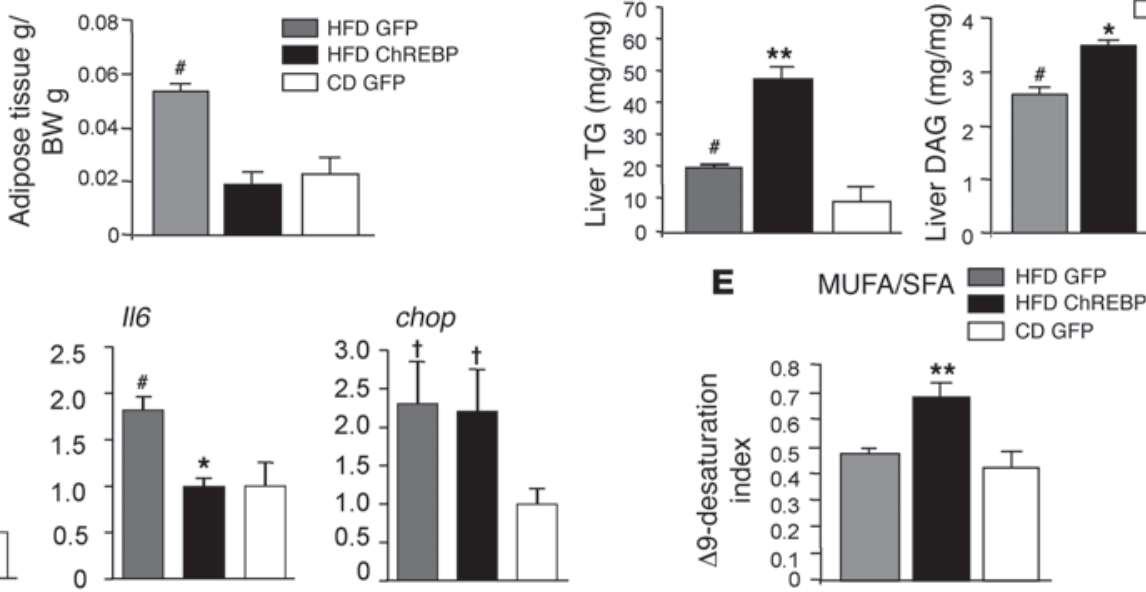

REBP

0

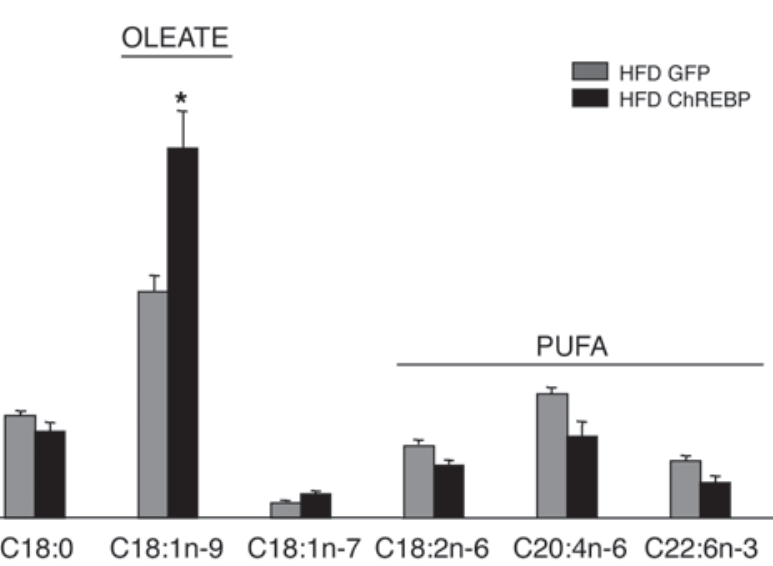

Figure 8

Exacerbated steatosis in HFD-fed ChREBP mice. Analyses were carried out in HFD-fed GFP and HFD-fed ChREBP mice, as described in the Methods section. GFP mice maintained on a standard CD for a similar number of weeks were used as controls. Mice were sacrificed in the fasted state. (A) Liver macroscopy and Oil Red O staining of liver sections (original magnification, $\times 40$ ). (B) Liver and adipose tissue weight (epididymal) (expressed as g/g of body weight [BW]). (C) Liver TGs and diacylglycerol concentrations. (D) qRT-PCR analysis of Tnfa, I/6, and chop. (E) Analysis of the $\Delta 9$-desaturation index (MUFA/SFA ratio). (F) Percentage of cellular fatty acid content. Nine lipid species (as indicated) were analyzed and used to assess the quality of the steatosis generated. Results are the mean $\pm \mathrm{SEM}(n=6-8 /$ group $)$. \#P<<0.05 HFD-fed GFP versus CD-fed GFP mice; ${ }^{*} P<0.05,{ }^{* *} P<0.01$ HFD-fed ChREBP versus HFD-fed GFP mice; ${ }^{\dagger} P<0.05,{ }^{\dagger t} P<0.01 \mathrm{HFD}$ versus CD.

markers of inflammation (Ccl4, Mrc2, and Mgl1) (ref. 43 and data not shown) was observed in livers of HFD-fed ChREBP mice compared with HFD-fed GFP mice. Interestingly, a significant decrease in Il6 expression was measured (Figure 8D). Altogether, our results suggest that the ChREBP-mediated hepatic steatosis did not aggravate the inflammation status. Importantly, lipidomic analysis revealed that MUFA content was significantly increased in livers of HFD-fed ChREBP mice compared with that in HFD-fed GFP mice, as evidenced by a significant higher desaturation index (Figure 8E). Notably, oleate (C18:1 n-9) enrichment in livers of HFD-fed ChREBP mice occurred at the expense of PALM (C16:0), the percentage of which was significantly decreased (Figure 8F).

Beneficial metabolic and physiologic effects of ChREBP overexpression in $H F D$-fed mice. We next examined whether hepatic Akt phosphorylation on Ser473 and on Thr308 could be improved by ChREBP overexpression under HFD feeding (Figure 9A). As we observed in vitro (Figures 4-6), ChREBP overexpression caused a substantial increase in SCD1 protein content (Figure 9A) and markers of its activity (Figure $8 \mathrm{E})$. In agreement with a significant state of insulin resistance, 
A Liver lysates

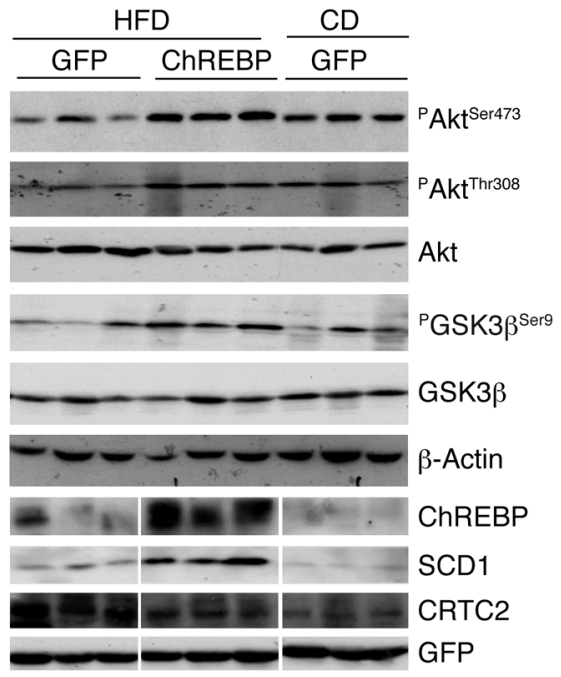

C Akt substrates (Ser/Thr)

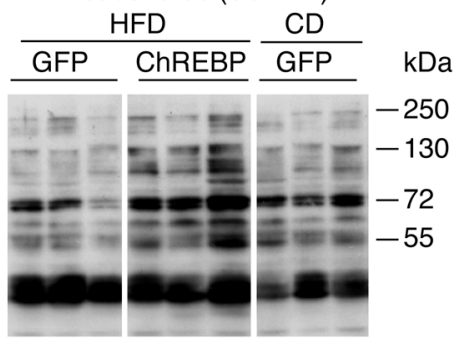

E Gluconeogenesis mRNA

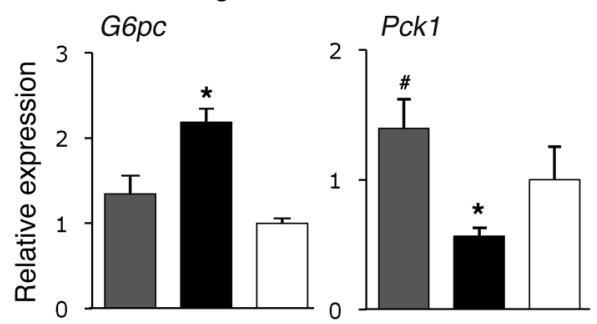

B
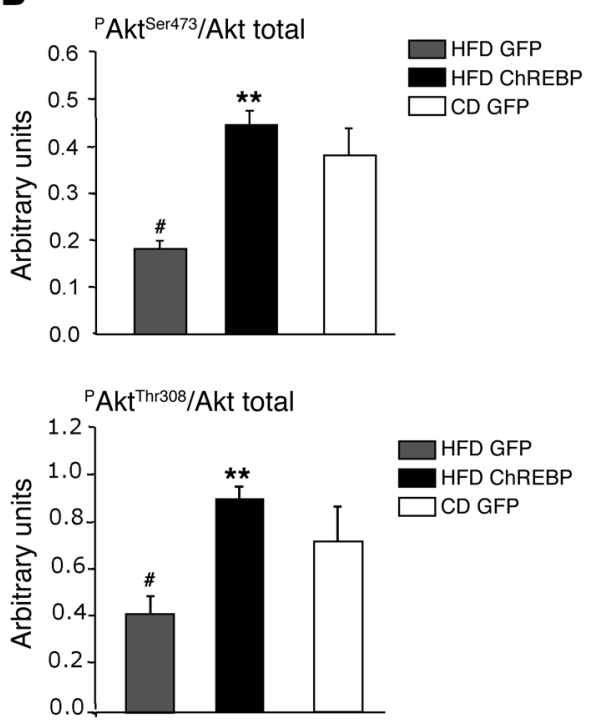

D

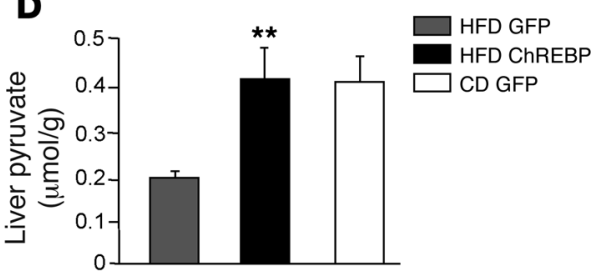

\section{Figure 9}

Improved Akt phosphorylation in livers of HFD-fed ChREBP mice. All analyses were carried out in HFD-fed GFP and ChREBP mice, as described in the Methods section. GFP mice maintained on a standard CD for a similar number of weeks were used as controls. Mice were sacrificed in the fasted state. (A) Ser473, Thr308 Akt phosphorylation, and Ser9 GSK3 $\beta$ phosphorylation in livers of overnight fasted CD-fed GFP, HFD-fed GFP, and HFD-fed ChREBP mice. Total protein content of Akt, GSK3 $\beta$ ChREBP, SCD1, CRTC2, and GFP are shown. Representative Western blots are shown. $\beta$-Actin was used as a loading control. Samples were run on the same gel, but lanes were not contiguous. (B) Quantification of the ratios of Ser473 and Thr308 Akt phosphorylation compared with total Akt protein content are shown. (C) Phosphorylation of Akt substrates (ranging from 55 to $250 \mathrm{kDa}$ ). A representative Western blot is shown. Samples were run on the same gel, but lanes were not contiguous. (D) Liver pyruvate concentrations. (E) qRT-PCR analysis of genes involved in the gluconeogenic pathway (G6pc, Pck1, Ppargc1a). Results are the mean \pm SEM ( $n=8-10$ /group). ${ }^{\#} P<0.05$ HFD-fed GFP versus CD-fed GFP mice; ${ }^{*} P<0.05$, ${ }^{* \star} P<0.01$ HFD-fed ChREBP versus HFD-fed GFP mice. the phosphorylation of Akt on Ser473 and Thr308 was reduced by about $50 \%$ in livers of HFD-fed GFP mice compared with that in CD-fed GFP mice (Figure 9B). Interestingly, Akt phosphorylation (Ser473 and Thr308) was not reduced in livers of HFD-fed ChREBP mice compared with that in HFD-fed GFP mice and was as high as in the control group (Figure 9, A and B). Accordingly, the phosphorylation on Ser/Thr residues of several Akt substrates (ranging from 250 to $55 \mathrm{kDa}$ ) was enhanced in HFD-fed ChREBP mice compared with that in HFD-fed GFP mice (Figure 9C). In addition, GSK3 $\beta$ phosphorylation on Ser9 was comparable in livers of HFD-fed ChREBP and CD-fed GFP mice (Figure 9A). Sustained phosphorylation in these key effectors of insulin signaling (namely Akt, Akt substrates, GSK3 $\beta$ ) occurred despite higher hepatic TG and DAG concentrations (Figure 8C). Fasting blood glucose concentrations were lower in HFD-fed ChREBP mice compared with those in HFDfed GFP mice (-30\%) (Table 1) and were correlated with a significant decrease in Pck1 (-60\%) and Ppargc1a gene expression (Figure 9E), and CRTC2 protein content (Figure 9A). Insulin regulates hepatic gluconeogenesis through the FoxO1-PPARGC1A interaction (44) and by promoting the phosphorylation and ubiquitin-dependent degradation of CRTC2 (45). The fact that the expression of several key components of the gluconeogenic pathway (namely PCK1, PPARGC1A, CRTC2) was decreased in livers of HFD-fed ChREBP mice compared with that in HFD-fed GFP mice is in favor of a rescued insulin-signaling pathway. However, it should be noted that G6pc gene expression, a known target gene of ChREBP (46), was 2-fold increased upon ChREBP overexpression (Figure 9E). Nevertheless, glucose tolerance was improved in HFD-fed ChREBP mice, and hyperinsulinemia was markedly decreased (Figure 10, A and B). As index of glycolytic flux in liver, hepatic pyruvate concentrations were measured (Figure 9D). Consistent with the transcriptional control exerted by ChREBP on L-PK expression (Figure 7A), a significant increase in hepatic pyruvate concentrations was observed (Figure 9D), supporting the idea that glucose flux is preferentially oriented toward glycolysis/lipogenesis in HFD-fed ChREBP mice.

Because $F g 21$ gene expression is under the transcriptional control of ChREBP (34) and because FGF21 has beneficial effects on plasma glucose and lipid profiles in diabetic rodents (47), mRNA and cir- 
A

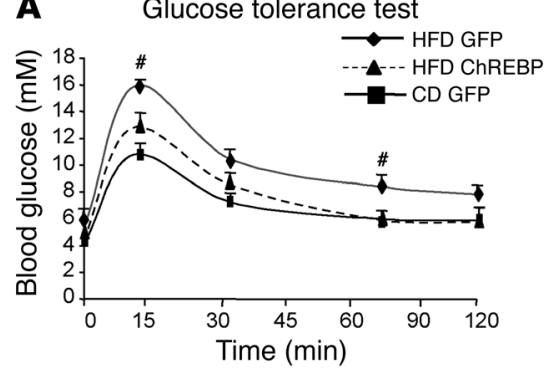

B

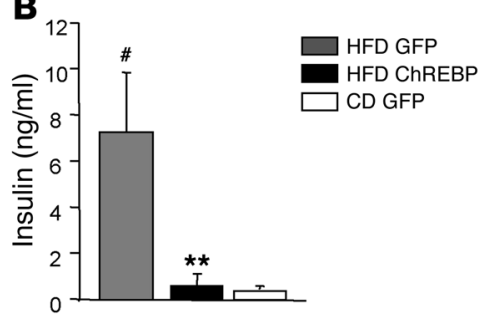

C

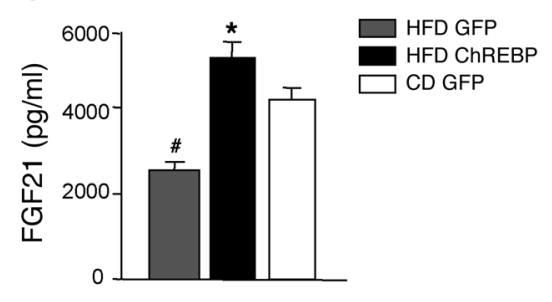

\section{Figure 10}

Improved glucose tolerance and insulin resistance in HFD-fed ChREBP mice. All analyses were carried out in HFD-fed GFP and ChREBP mice, as described in the Methods section. GFP mice maintained on a standard CD for a similar number of weeks were used as controls. (A) Glucose tolerance tests (1 g/kg) were performed in GFP and ChREBP mice. (B) Fasting insulin concentrations. (C) Fasting FGF21 concentrations. Results are the mean \pm SEM ( $n=8-10 /$ group). ${ }^{\#} P<0.05$ HFD-fed GFP versus CD-fed GFP mice; ${ }^{*} P<0.05,{ }^{* *} P<0.01$ HFD-fed ChREBP versus HFD-fed GFP mice.

culating levels of Fgf21 were measured (Figure 7B and Figure 10C). ChREBP overexpression led a to significant increase in Fgf 21 mRNA concentrations and circulating levels, raising the possibility that enhanced circulating FGF21 levels may have also contributed to the observed improvement in glucose tolerance (Figure 10A).

ChREBP expression is positively related to the degree of hepatic steatosis and inversely to insulin resistance in patients with NASH. In order to translate the findings obtained in mice to human pathology, 25 patients with histologically proven NASH were studied (48). Individual characteristics of patients are listed in Table 2. Mean BMI was $31 \pm 5.5 \mathrm{~kg} / \mathrm{m}^{2}$, and most patients had features of the metabolic syndrome. Mean homeostasis model assessment (HOMA) was $5.6 \pm 2.6$. Mean percentage of steatosis was $55 \% \pm 21 \%$, and, among the 25 patients, $28 \%$ had severe fibrosis (F3, F4). Only $16 \%$ had no fibrosis. The diagnosis of NASH was defined as steatosis of more than $20 \%$ of the liver and the presence of either hepatocyte ballooning or intralobular hepatocyte necrosis. Using the median values of steatosis (50\%) and the HOMA index (4.8), CHREBP mRNA levels were 2 -fold higher in patients with steatosis of more than $50 \%$ compared with those in patients with steatosis of less than $50 \%(0.33 \pm 0.03$ relative expression [SEM] vs. $0.16 \pm 0.03$ relative expression [SEM]; $P=0.04$ ) (Figure 11A). Conversely, ChREBP expression was decreased by $50 \%$ in patients with a HOMA index value of more than 4.8 compared with patients with a HOMA index value of less than $4.8(0.18 \pm 0.06$ relative expression [SEM] vs. $0.35 \pm 0.13$ relative expression $[\mathrm{SEM}] ; P=0.04$ ) (Figure $11 \mathrm{~B}$ ). Finally, significant correlations were found between the HOMA index $\left(r^{2}=0.25 ; P=0.03\right)$ (Figure $\left.11 \mathrm{C}\right)$ or the degree of liver steatosis $\left(r^{2}=0.27 ; P=0.02\right)$ (Figure 11D) and CHREBP mRNA levels in livers of these patients.

\section{Discussion}

NAFLD is an increasing health concern now considered as a component of the metabolic syndrome (1). Excessive accumulation of TGs in hepatocytes is the hallmark of NAFLD. Dysregulated lipogenesis has been shown to contribute to the pathogenesis of hepatic steatosis in both humans $(16,49)$ and rodents $(50)$. However, the contribution of the glucose-sensitive lipogenic transcription factor ChREBP to the pathophysiology of human steatosis and/or insulin resistance has not been clearly addressed. In addition, despite the widely accepted association between hepatic steatosis and insulin resistance, it remains unclear whether a causal relationship always exists. Our study reports that ChREBP overexpression in mouse liver leads to a significant increase in hepatic TG concentrations and hepatic steatosis development. Interestingly, ChREBP overexpression in livers of HFD mice, while inducing a larger hepatic lipid overload, was protective against liver insulin resistance and was correlated with beneficial lipid species accumulation (i.e., oleate, C18:1 n-9, MUFA) and decreased percentage of PALM (C16:0). To our knowledge, our work is the first to report that hepatic ChREBP expression varies positively according to the degree of steatosis and inversely to insulin resistance in a cohort of patients with NASH.

ChREBP is a basic helix-loop-helix/leucine zipper transcription factor, playing a critical role in hepatic fatty acid synthesis (25-27). To further address the importance of ChREBP in the control of de novo lipogenesis and its relationship to insulin resistance development, we chose to overexpress a dephosphorylated isoform of ChREBP in mouse liver. Although it was previously reported that mutations of Ser196 and/or Thr666 may not result in a constitutively active form of ChREBP (51), we report here that ChREBP target genes were efficiently induced under both substrate abundance (standard CD and HFD) and fasting conditions. Indeed, ChREBP overexpression induced, the entire lipogenic/esterification program in liver independently of an increase in SREBP-1c activity and/or LXR $\alpha$ expression. Aside from previously known targets of ChREBP, such as L-PK, ACACA, FASN, GPAT, G6PC, and FGF21 $(19,34,52)$, our results reveal that SCD1 and Elovl6 are key ChREBP target genes. Indeed, a selective enrichment in their lipid products (i.e., MUFA) (Figure 2G) was observed upon ChREBP overexpression in liver under both standard and HFDs. Interestingly, both SCD1 and Elovl6 were identified among 700 other direct target genes of ChREBP in a genome-wide scale analysis performed in HepG2 cells using ChIP sequencing (53).

Altogether, our results strongly support the hypothesis that ChREBP favors TG accumulation and may in fact protect liver from lipotoxicity by buffering the accumulation of detrimental fatty acids. In mammalian cells, fatty acids are required for the synthesis of phospholipid components of the membrane and energy storage as TGs. However, over accumulation of fatty acids can be considered toxic/deleterious $(14,54)$. To prevent lipotoxicity, cells can enhance incorporation of fatty acids into TGs that are then stored into lipid droplets (55). Under HFD feeding conditions (in terms of energy, $60 \%$ coming from fat), enhancing 


\section{Table 2}

Clinical and biochemical characteristics of patients with NASH evaluated in this study

$\begin{array}{lc}\text { Variables } & \text { Mean } \pm \text { SD } \\ \text { Age }(\mathrm{yr}) & 55 \pm 11 \\ \text { Sex ratio (male/female) } & 17 / 8 \\ \text { BMI }\left(\mathrm{kg} / \mathrm{m}^{2}\right) & 31 \pm 5.5 \\ \text { Waist }(\mathrm{cm}) & 107 \pm 11 \\ \text { Diabetes }(n) & 7 \\ \text { High blood pressure }(n) & 11 \\ \text { ALT (IU/l) } & 77 \pm 40 \\ \text { AST (IU/l) } & 52 \pm 29 \\ \gamma \text { GT (IU/l) } & 100 \pm 11 \\ \text { Cholesterol (mmol/l) } & 6.6 \pm 1.2 \\ \text { HDL cholesterol (mmol/l) } & 1.6 \pm 0.36 \\ \text { TGs (mmol/l) } & 2.2 \pm 1.4 \\ \text { Insulin }(I U / m l) & 20.1 \pm 8.2 \\ \text { Glucose (mmol/l) } & 5.9 \pm 1.8 \\ \text { HOMA } & 5.6 \pm 2.6 \\ \text { Steatosis (\%) } & 55 \pm 21 \\ \text { NAS } & 4.4 \pm 1.4 \\ \text { Fibrosis stage } & \\ \text { F0 (\%) } & 16 \\ \text { F1 (\%) } & 8 \\ \text { F2 (\%) } & 48 \\ \text { F3 (\%) } & 24 \\ \text { F4 (\%) } & 4 \\ & \end{array}$

After a 12-hour overnight fast, venous blood samples were drawn to determine levels of blood glucose and ALT concentrations. Insulin resistance was assessed using the HOMA index as follows: fasting insulin $(\mathrm{mU} / \mathrm{l}) \times$ fasting plasma glucose $(\mathrm{mmol} / \mathrm{l}) / 22.5(90)$.

endogenous generation of fatty acids via ChREBP overexpression had clear beneficial effects on both hepatic insulin signaling and glucose metabolism. Genes involved in lipogenesis and TG levels were further enhanced $(+125 \%)$, and a notable switch in cellular fatty acid percentage was observed compared with HFD-fed GFP conditions. ChREBP overexpression induced hepatic steatosis with a greater accumulation of oleate than PALM (45\% and $15 \%$, respectively), compared with GFP HFD steatosis, in which the oleate and PALM levels were nearly equivalent (27\% and $24 \%$, respectively) (Figure 12). Our results support the novel concept of lipoexpediency (in opposition to lipotoxicity) recently proposed by Semenkovich and colleagues (13). Emerging data implicate de novo lipogenesis in the generation of beneficial lipid transmitters that reduce disease risk (14). Although it may seem somehow paradoxical, optimizing lipid signals generated by de novo lipogenesis could help redirecting fat toward benefit, even in the setting of lipid overload (13). According to this hypothesis, it was reported that overexpression of DGAT1 improves storage of neutral TG within lipid droplets, thereby reducing deleterious lipid species, inflammation, and insulin resistance in macrophages and/or muscle cells (56-58). Disruption of elongases, enzymes that are crucial for fatty acids chain length (59), leads to severe metabolic alterations by changing free fatty acids pools (60), while their overexpression increases MUFA accumulation and TG synthesis and storage in lipid droplets. Lastly, the relevance of desaturase activity (namely SCD1, which introduces a cis double bond in the $\Delta 9$ position of saturated 16- and 18-carbon fatty
acyl-CoA substrates; ref. 35) in fatty acid partitioning has recently been recognized as important as shown in experiments in which knockdown of unsaturase, by decreasing the MUFA/SFA ratio, causes ER stress and apoptosis. Of note, the expression of Dgat1, Elovl6, and Scd1 was significantly increased upon ChREBP overexpression in liver under HFD conditions (Figure 7A).

In fact, our results suggest that the lipogenic SCD1 enzyme was instrumental in the beneficial effects mediated by ChREBP overexpression. By stimulating SCD1 expression in liver, ChREBP led to the subsequent rise in oleate (C18:1n-9) concentrations under $\mathrm{CD}$ and HFD feeding. Experiments performed in primary hepatocytes revealed that ChREBP or SCD1 overexpression and/or oleate incubation prevented the deleterious effect of PALM on Akt phosphorylation (Ser473 and Thr308) and on chop induction. Our in vitro experiments support studies showing that lipotoxicity and ER stress are almost exclusively attributed to SFA $(6,39)$ and that they can be prevented by oleate and/or palmitoleate incubation $(61,62)$. ChREBP overexpression, through its positive control of SCD1, allowed for the conversion of PALM in the culture medium into specific lipids that are beneficial for insulin sensitivity and for the prevention of ER stress (i.e., chop induction). These results reinforce the concept that TG synthesis is crucial to prevent harmful effects on organelles and/or cell signaling. In agreement with this hypothesis, when SCD1 was inhibited pharmacologically or through a shRNA strategy, the protective effect of ChREBP on insulin signaling was significantly attenuated. Surprisingly, while it has been reported that SCD1 knockdown causes ER stress by causing SFA accumulation (39), no significant reinduction of chop was observed when SCD1 was silenced in the context of ChREBP/ PALM conditions, suggesting that ChREBP overexpression alone could be sufficient to overcome the deleterious effect of PALM on chop induction. The contribution of SCD1 to hepatic steatosis, insulin resistance, and/or inflammation is rather complex. On one hand, inactivation of SCD1 in liver prevents fatty liver, insulin resistance, and obesity induced by high carbohydrate feeding (63) but, on the other hand, worsens diabetes in $o b / o b$ mice (64). Furthermore, SCD1 deficiency aggravates the inflammatory state under conditions of methionine choline-deficient diet and in response to dextran sulfate sodium-induced acute colitis, causing hepatic overaccumulation of SFA that triggers hepatocellular apoptosis, liver damage, and NASH (65). Interestingly, oleic acid feeding and in vivo SCD1-rescued expression alleviates the dextran sulfate sodium-induced phenotype, suggesting that SCD1 and its related lipid species may serve as potential targets for intervention and/or treatment of inflammatory diseases (66). Therefore, consumption of an oleic-enriched diet or selective modulation of the lipogenic pathway via ChREBP and/or SCD1 could represent an interesting strategy to ameliorate and/or buffer the toxic effect of SFAs by diverting them to safe forms of lipids. In agreement with this concept, a recent study reports that consumption of an oleicenriched diet improves insulin sensitivity and decreases inflammation in muscles from aged rats (67).

Altogether, our study reveals that HFD-fed ChREBP mice are metabolically healthier than their controls, showing reduced fasting blood glucose, improved glucose tolerance, and reduced hyperinsulinemia, despite a higher grade of hepatic steatosis. It should be noted that ChREBP-mediated steatosis was dissociated from insulin resistance, despite elevated concentrations of DAG (a 2 -fold increase under CD but only a $20 \%$ under HFD), an important lipid intermediate previously implicated in hepatic insulin 
A
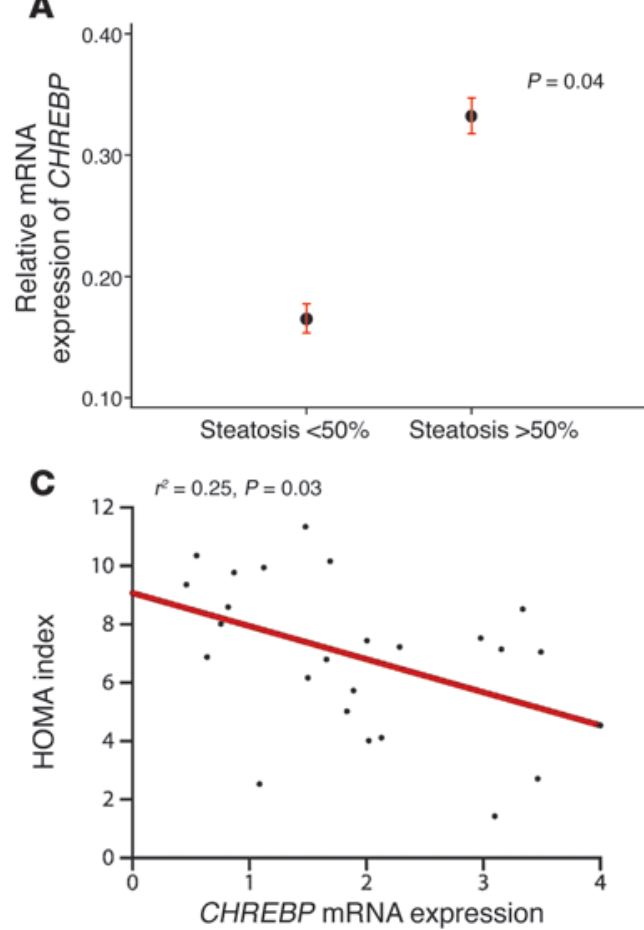

B
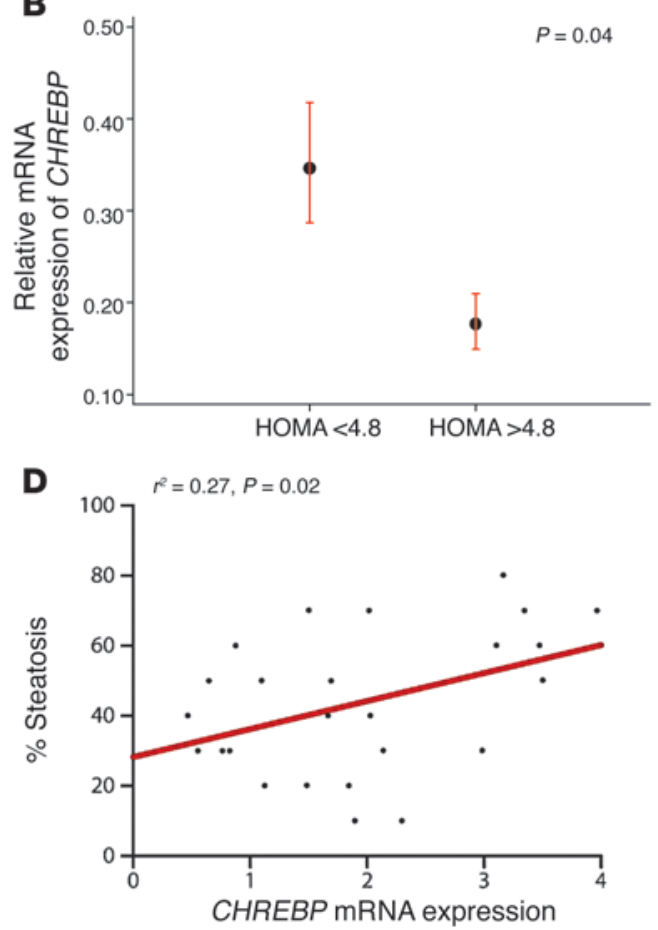

Figure 11

ChREBP expression is positively related to the degree of hepatic steatosis and inversely to insulin resistance in patients with $\mathrm{NASH}$. $\mathrm{CHREBP}$ mRNA levels in livers of fasted patients with histologically proven NASH. Histologic diagnosis of NASH was defined as steatosis of more than $20 \%$ and the presence of either hepatocyte ballooning or intralobular hepatocyte necrosis (48). (A) qRT-PCR analysis of $C H R E B P$ in livers of patients with steatosis under $(n=12)$ or above $50 \%(n=13)$. (B) qRT-PCR analysis of $C H R E B P$ in livers of patients with HOMA index values of less than $4.8(n=12)$ versus those with HOMA index values of more than $4.8(n=13)$. (C) Correlations between the HOMA index $\left(r^{2}=0.25 ; P=0.03\right)$ or $(D)$ the degree of steatosis $\left(r^{2}=0.27 ; P=0.02\right)$ and ChREBP expression in livers of these patients were performed. Symbols represent individual patients.

resistance in both rodents $(31,32)$ and humans (8). Of note, our results are consistent with other mouse models in which increased DAG concentrations were not associated with insulin resistance. In these studies, overexpression of DGAT2 in liver (9) or blockade of VLDL secretion mediated by MTP inactivation (68) resulted in a clear dissociation between hepatic steatosis and insulin resistance, despite a significant increase in hepatic DAG and ceramide concentrations. This discrepancy might be explained if different pools of DAG and ceramide exist within the hepatocytes, as previously suggested in muscle cells (69), with only certain pools being able to regulate inhibitors of insulin signaling (70). Another important feature of ChREBP overexpression is that amplified fat storage occurred in liver but was reduced in white adipose depot (under both standard diet and HFD). As a result, serum NEFAs, which predominantly arise from adipose tissue lipolysis at the fasting state, were reduced in HFD-fed ChREBP mice. Altogether, our current results contrast with previous studies reporting that ChREBP deficiency improves hepatic steatosis and insulin resistance in leptin-deficient $o b / o b$ mice $(25,27)$. As mentioned in the Introduction, paradoxical metabolic/physiologic consequences of ChREBP deficiency have been previously reported, since deleterious or beneficial phenotypes can be observed depending whether ChREBP deficiency is generated under a context of lipid overload. We show here that ChREBP overexpression dissociated hepatic steatosis from insulin resistance by modifying the MUFA/SFA bal- ance. Our results also suggest that enhanced glycolytic rates mediated by ChREBP overexpression (at the level of L-PK, evidenced by increased hepatic pyruvate concentrations) also contributed to the improvement of glucose tolerance in HFD-fed ChREBP mice (Figure 9D). Indeed, it has been previously reported that enhancing glycolytic rates through GCK in obese mice had beneficial influences on overall whole-body energy balance, including glucose tolerance (71). The fact that ChREBP global deficiency leads to decreased glycolytic rates (as evidenced by a lower pyruvate/phosphoenolpyruvate ratio) as well as major alterations in energy substrate utilization also supports this hypothesis. Lastly, the human homolog of ChREBP, WBSCR14 (also known as Mon$\mathrm{doB})$, is located on a chromosomal region deleted in the WilliamsBeuren syndrome, a syndrome characterized in $75 \%$ of the cases by severe glucose intolerance (72). Altogether, our results suggest that ChREBP could act as a dual regulator of glycolysis and gluconeogenesis, which have a reciprocal relationship in response to glucose. Indeed, under both standard diets and HFDs, key components of gluconeogenesis (namely PCK1, PPARGC1A, FOXO1, CRTC2) were decreased upon ChREBP overexpression. Interestingly, we observed a substantial decrease in the expression of the deacetylase SIRT1 in livers of HFD-fed ChREBP mice compared with that in HFD-fed GFP mice (data not shown), confirming the ChREBP-mediated inhibition of SIRT1 (73). In the liver, SIRT1 controls gluconeogenic activity by modulating the acetylation 


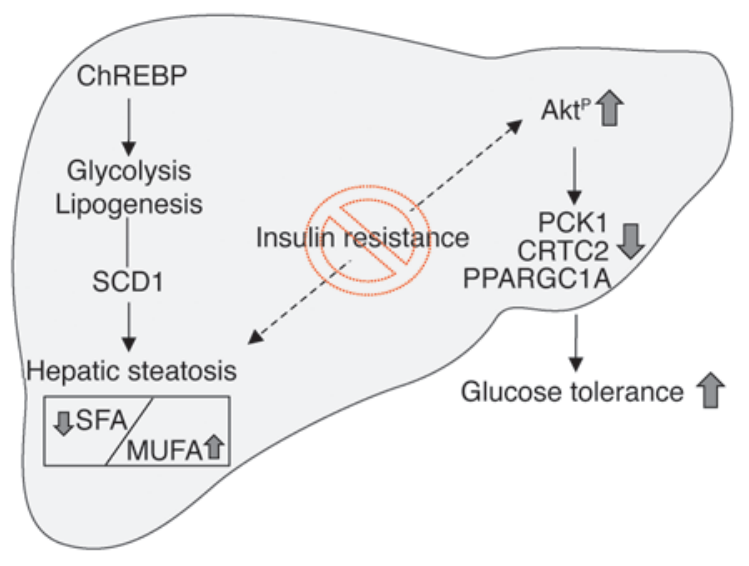

Figure 12

Antilipotoxic effect of ChREBP overexpression in mouse liver. Upon ChREBP overexpression in livers of mice fed a HFD, glycolysis and lipogenesis are stimulated, leading to the development of hepatic steatosis. ChREBP overexpression by stimulating SCD1 activity leads to a modification in lipid composition (namely a change in the monounsaturated [MUFA] to saturated [SFA] ratio) and dissociates hepatic steatosis from insulin resistance. Increased phosphorylation of Akt (Ser473 and Thr308) and of downstream substrates was measured in HFD-fed ChREBP mice, and glucose tolerance was improved. Our results provide insights into the causal relationship between hepatic steatosis and insulin resistance and demonstrate that increasing the lipogenic pathway may protect against insulin resistance by raising beneficial lipid species. CRTC2, CREB-regulated transcription coactivator 2; PPARGC1A, peroxisome proliferator-activated receptor $\gamma$ coactivator 1- $\alpha$; PCK1, phosphoenolpyruvate carboxykinase.

levels of a network of transcription factors and/or coactivators, including PPARGC1A (74-76). The deacetylation of PPARGC1A is tightly linked with enhanced PPARGC1A transcriptional activation (77). Therefore, as previously suggested by Noriega and colleagues (73), the ChREBP-mediated repression of SIRT1 could inhibit energy-producing pathways, such as gluconeogenesis (notably through increased acetylation levels of PPARGC1A). In agreement with this hypothesis, acetylation levels of PPARGC1A were decreased by $50 \%$ in livers of ChREBP knockout mice (73). Lastly, since the recent ChIP sequencing analysis of ChREBP identified gluconeogenic genes as direct targets of ChREBP (53), it is possible that the effect observed on these genes could be a direct consequence of ChREBP transcriptional repression rather than an indirect effect of increased Akt signaling.

Fatty acid biosynthesis is thought to occur to a greater extent in rodents compared with humans and could be even considered as a minor contributor to whole body lipid stores in present-day humans eating a typical HFD. However, Donnelly et al. (16) previously estimated that close to $30 \%$ of TGs accumulated in livers of patients with NAFLD came from de novo lipogenesis, and another study which reevaluated the contribution of lipogenesis to the development of hepatic steatosis showed that the expression of fatty acid metabolism-related genes, such as ACACA and FASN, is significantly increased in NAFLD (78). Interestingly, ChREBP expression (mRNA and protein content) was recently found to be elevated (a 2 -fold increase) in livers of obese patients (79). To our knowledge, our study is the first to report increased ChREBP expression in patients with NASH. In addition, the 50\% decreased ChREBP expression in livers of patients with severe insulin resis- tance (HOMA > 4.8) reinforces the concept that ChREBP-mediated steatosis is not necessarily related to a high degree of insulin resistance. In our population, insulin resistance, as assessed by the HOMA index, was not correlated (positively or negatively) with steatosis $\left(r^{2}=0.03 ; P=0.4\right)$. This differs from other studies that have shown a relationship between insulin resistance and the extent of steatosis. However, several findings indicate that this association is complex and sometimes absent due to alterations of some specific proteins, such as ATGL or adiponutrin (PNPLA3), or to insulin resistance at the receptor level (80). It is important to consider that the HOMA index is testing insulin resistance for the glucose pathway (i.e., in the fasting state, hepatic glucose production). In contrast, steatosis is indicative of the persistent effect of insulin on the lipogenic pathway (de novo lipogenesis) in the liver (81) and of adipose tissue resistance, leading to increased levels of NEFA and lipoprotein remnants being captured by the liver. In this setting, the absence of a positive relation between insulin resistance and steatosis could indicate the complexity of the relationships between insulin resistance and liver lipid accumulation. In addition, it has been recently suggested that the type of fatty acids that are stored in the liver could be more relevant than the degree of steatosis regarding the mechanisms of fatty liver injuries $(5,54)$. The absence of correlation between insulin resistance and steatosis does not preclude a possible relation between the level of hepatotoxic fatty acids and insulin resistance. Lastly, although it would have been highly relevant, we were not able to perform lipidomic analysis in this study, mostly because of methodological and ethical limitations to sampling human liver tissue.

By unraveling the contribution of lipogenesis via ChREBP to the development of hepatic steatosis in mice and in patients with NASH, our study shows that fatty acid composition is an important determinant of insulin sensitivity and, more importantly, that hepatic steatosis can occur independently of insulin resistance. Our results provide insights which we believe to be novel into the causal relationship between hepatic steatosis and insulin resistance and demonstrate that increasing the lipogenic pathway via ChREBP may protect against insulin resistance by raising beneficial lipid species. By combining experimental and human data, we believe that our study also provides original insight on the complex pathogenesis of NAFLD.

\section{Methods}

Animals and diets. Seven- to eight-week-old male C57BL/6J mice were purchased from Charles River and adapted to the environment for 2 weeks before study. All mice were housed in colony cages with a 12-hour-light/ 12-hour-dark cycle in a temperature-controlled environment (the dark cycle going from 3:00 am until 3:00 pm). Mice had free access to water and standard CD (in terms of energy, 65\% from carbohydrate, 11\% from fat, and $24 \%$ from protein). For HFD studies, C57BL/6J mice (Charles River) were maintained on a HFD (EF R/M acc. D12492, Ssniff Spezialdiaten $\mathrm{GmbH}$, modified upon personal request) for a total of 10 weeks, starting at 4 weeks of age. Modified HFD consisted of crude nutrients (fat 35\% [SFA $20 \%$, MUFA $12 \%$, cholesterol $3 \%$ ] sugar/dextrin $22 \%$ ) (in terms of metabolized energy, $60 \%$ from fat, $21 \%$ from carbohydrate and $19 \%$ from protein). Six weeks after the beginning of the HFD, mice were injected with 1 single dose of $5 \times 10^{9}$ pfu of GFP or ChREBP adenovirus (as described below) and maintained on HFD for another 4 weeks. Mice injected with a similar dose of GFP and maintained for 10 weeks on standard CD were used as controls. All studies were performed on 14-week-old mice, either fasted 18 hours or under fed conditions as stated in Table 1. For "fed" conditions, mice were 
sacrificed between 9:00 and 10:00 am (during dark cycle). For "fasting" conditions, mice were fasted overnight (12 hours).

Generation of the ChREBP adenovirus construct. The full-length wild-type ChREBP-z isoform mutated on Ser196 and Thr666 (19) was subcloned into the shuttle vector pAd Track-CMV. Recombinant adenoviral constructs ChREBP and GFP recombinant adenovirus were produced in HEK293T cells and purified on cesium chloride gradients before use by the Laboratoire of Thérapie Génique.

Injection of the ChREBP adenovirus and in vivo insulin stimulation. Male mice were anesthetized with isoflurane before injection through the penis vein with $5 \times 10^{9} \mathrm{pfu}$ of either GFP or ChREBP in a final volume of $200 \mu \mathrm{l}$ of sterile $\mathrm{NaCl} 9 \%$. Experiments and analysis were performed 4 weeks after the adenoviral injection (a single dose). For insulin signaling experiments, mice were fasted overnight and then injected with $\mathrm{NaCl} 9 \%$ or 1 unit of regular human insulin $/ \mathrm{kg}$ (Actrapid Penfill, NovoNordisk) via the portal vein. Three minutes after injection of the insulin bolus, livers were removed and snap frozen in liquid nitrogen.

Glucose, insulin, and pyruvate tolerance tests. Glucose tolerance tests were performed 4 weeks after adenoviral injections by glucose gavage (1 g D-glucose $/ \mathrm{kg}$ body weight) after an overnight fast. Insulin tolerance tests were performed by intraperitoneal injection of human regular insulin ( 0.75 unit insulin/kg body weight; Actrapid Penfill, NovoNordisk) 5 hours after food removal. Pyruvate tolerance tests were performed by intraperitoneal injection of pyruvate (monosodium salt, Boehringer Mannheim) $(2 \mathrm{~g} / \mathrm{kg}$ body weight) after an overnight fast. Blood glucose was determined using the One-Touch AccuChek Glucometer (Roche).

Analytical procedures. Serum concentrations of TG, NEFA, AST, and ALT were determined using an automated Monarch device (Laboratoire de Biochimie, Faculté de Médecine Bichat, Paris, France). Serum insulin concentrations were determined using a rat insulin ELISA Assay Kit (Crystal Chem) using a mouse insulin standard. FGF21 concentrations were measured using the Rat/ Mouse FGF-21 ELISA Kit (EZRMFGF21-26K, Millipore). Liver G6P, glycogen concentrations, and pyruvate concentrations were determined as previously described (82). Liver TGs were measured with a colorimetric diagnostic kit according to the manufacturer's instructions (Triglycerides FS, Diasys).

Staining techniques. For histology studies, livers were fixed in $4 \%$ neutral buffered formalin and embedded in paraffin. Then, $7-\mu \mathrm{M}$ sections were cut and stained with H\&E. For the detection of neutral lipids, liver cryosections were stained using the Oil Red O technique (83) using $0.23 \%$ dye dissolved in 65\% isopropyl alcohol for 10 minutes.

Primary cultures of hepatocytes. Mouse hepatocytes were prepared as described previously (17) and cultured in the presence of $5 \mathrm{mM}$ glucose and $0.48 \mathrm{mM}$ albumin-bound PALM (C16:0) at a fatty acid/albumin ratio of $4: 1$ for 24 hours or $0.48 \mathrm{mM}$ albumin-bound PALM/oletate $(0.3 \mathrm{mM}$ each) (OLE/PALM). The source of albumin used was fatty acid-free bovine serum albumin (Sigma-Aldrich). After 24 hours in these different conditions, hepatocytes were infected with 3 pfu per cell of GFP, ChREBP, SCD1, or shSCD1 (GeneCust) for 24 hours. After the adenofection period, insulin $(1 \mathrm{nM})$ was added to the culture medium for times ranging from 0 to 5 minutes. The SCD1 inhibitor (SCD1 ${ }^{\text {inhib}) ~(r e f ~ n o . ~ A 939572, ~ Q S C h e m) ~}$ (36) was used for 24 hours at a final concentration of $10 \mathrm{nM}$.

Measurement of glucose production in primary hepatocytes. After specific adenofection (GFP or ChREBP), hepatocytes were incubated with cell culture medium containing no glucose (G0) but containing lactate and pyruvate substrates with BSA or PALM $(0.48 \mathrm{mM})$ plus or minus insulin $(100 \mathrm{nM})$ for 24 hours. Hepatic glucose production was measured after PCA neutralization as previously described (84).

Lipidanalysis. Lipid assays were performed by the lipidomic facility (Bio-Medical Federative Research Institute of Toulouse, INSERM, Plateforme MetaToul). Briefly, after homogenization of tissue samples in methanol $/ 5 \mathrm{mM}$
EGTA $(2: 1 \mathrm{v} / \mathrm{v})$, lipids corresponding to an equivalent of $1 \mathrm{mg}$ of tissue were extracted in chloroform/methanol/water $(2.5: 2.5: 2.1, \mathrm{v} / \mathrm{v} / \mathrm{v})$, in the presence of internal standards: 1,3-dimyristine (for DAG) and glyceryl triheptadecanoate (for TG). Chloroform phases were evaporated to dryness. Neutral lipids were purified over an SPE column (Macherey Nagel glass Chromabond pure silice, $200 \mathrm{mg}$ ): after washing cartridge with $2 \mathrm{ml}$ chloroform, lipid extract was applied on the cartridge in $20 \mu \mathrm{l}$ chloroform, and neutral lipid were eluted with chloroform/methanol (9:1, v/v; $2 \mathrm{ml}$ ). The organic phase was evaporated to dryness and dissolved in $20 \mu \mathrm{l}$ ethyl acetate. A sample $(1 \mu \mathrm{l})$ of the lipid extract was analyzed by gas-liquid chromatography on a FOCUS Thermo Electron system, using Zebron-1 (Phenomenex) fused silica capillary columns $(5-\mathrm{m} \times 0.32-\mathrm{mm}$ inside diameter [i.d.], $0.50-\mu \mathrm{m}$ film thickness) (85). Oven temperature was programmed from $200^{\circ} \mathrm{C}$ to $350^{\circ} \mathrm{C}$ at a rate of $5^{\circ} \mathrm{C}$ per minute, and the carrier gas was hydrogen (7.25 psi). The injector and the detector were at $315^{\circ} \mathrm{C}$ and $345^{\circ} \mathrm{C}$, respectively.

To measure ceramide and sphingomyelin, total lipids corresponding to $2 \mathrm{mg}$ of tissue were extracted according to the method of Bligh and Dyer in chloroform/methanol/water $(2.5: 2.5: 2.1, \mathrm{v} / \mathrm{v} / \mathrm{v})$ in the presence of the internal standards ceramide NC15 (2 $\mu$ g, prepared according to ref. 86); the dried lipid extract was submitted to a mild alkaline treatment in methanolic $\mathrm{NaOH} 0.6 \mathrm{~N}(1 \mathrm{ml})$ and then to silylation in $50 \mu \mathrm{l}$ BSTFA (1\%TMSCl)/acetonitrile $(1: 1, v / v)$ overnight at room temperature. Sample $(5 \mu \mathrm{l})$ was directly analyzed by gas-liquid chromatography (4890 Hewlett Packard system, using a RESTEK RTX-50 fused silica capillary columns, $30-\mathrm{m} \times 0.32-\mathrm{mm}$ i.d., $0.1-\mu \mathrm{m}$ film thickness). Oven temperature was programmed from $195^{\circ} \mathrm{C}$ to $310^{\circ} \mathrm{C}(12$ minutes $)$ at a rate of $3.5^{\circ} \mathrm{C}$ per minute, and the carrier gas was hydrogen $(7.25 \mathrm{psi})$. The injector and the detector were at $310^{\circ} \mathrm{C}$ and $340^{\circ} \mathrm{C}$, respectively.

To measure total hepatic fatty acid methyl ester (FAME) molecular species, lipids corresponding to an equivalent of $1 \mathrm{mg}$ of liver were extracted in the presence of glyceryl triheptadecanoate $(0.5 \mu \mathrm{g})$ as an internal standard. The lipid extract was transmethylated with $1 \mathrm{ml}$ of BF3 in methanol (14\% solution; Sigma-Aldrich) and $1 \mathrm{ml}$ of hexane for 60 minutes at $100^{\circ} \mathrm{C}$ and evaporated to dryness, and the FAMEs were extracted with hexane/water $(2: 1)$. The organic phase was evaporated to dryness and dissolved in $50 \mu \mathrm{l}$ ethyl acetate. A sample $(1 \mu \mathrm{l})$ of total FAME was analyzed by gas-liquid chromatography (Clarus 600 Perkin Elmer system, with Famewax RESTEK fused silica capillary columns, $30-\mathrm{m} \times 0.32-\mathrm{mm}$ i.d., $0.25-\mu \mathrm{m}$ film thickness). Oven temperature was programmed from $110^{\circ} \mathrm{C}$ to $220^{\circ} \mathrm{C}$ at a rate of $2^{\circ} \mathrm{C}$ per minute, and the carrier gas was hydrogen $(7.25 \mathrm{psi})$. The injector and the detector were at $225^{\circ} \mathrm{C}$ and $245^{\circ} \mathrm{C}$, respectively.

To measure hepatocyte fatty acid composition, cells were washed twice with ice-cold PBS and scraped into PBS. After centrifugation, the cell pellet was resuspended in PBS and sonicated. Cellular lipids were extracted with hexane/isopropanol $(3: 2, \mathrm{v} / \mathrm{v})$ prior to transmethylation with $1 \mathrm{ml}$ of BF3 in methanol (14\% solution; Sigma-Aldrich) and $1 \mathrm{ml}$ of hexane for 60 minutes at $100^{\circ} \mathrm{C}$. FAMEs were then extracted with hexane and analyzed by gas-liquid chromatography. To assess the effect of SCD1 inhibitor, we estimated the $\triangle 9$-desaturation index as the abundance of SCD1 products (oleic and palmitoleic acids) relative to both SCD1 products and substrates (palmitic and stearic acids).

Isolation of total $m R N A$ and analysis by $q R T-P C R$. Total cellular RNA was extracted using the RNeasy Kit (Qiagen), and 500 ng of RNA were reverse transcribed. qRT-PCR analysis was performed with a LightCycler instrument (Roche Applied Science) and SYBR green detection of amplified products. Primers for Gck, Pklr, Acaca, Fasn, Scd1, GPAT, Pck1, ChREBP, SREBP-1c (17, 27), LXR (23), Pparg (87), and Fgf21 (33) were previously described. Other primers were as follows: Elovl6 (sense 5'-ACAATGGACCTGTCAGCAAA-3'; antisense 5'-GTACCAGTGCAGGAAGATCAGT-3'); Dgat1 
(sense 5'-TTCCGCCTCTGGGCATT-3'; antisense 5'-AGAATCGGCCCACAATCCA-3'); Dgat2 (sense 5'-AGTGGCAATGCTATCATCATCGT-3'; antisense $5^{\prime}$-AAGGAATAAGTGGGAACCCAGATCA-3'); MTP (sense 5'TGGACGTTGTGTTACTGTGG-3'; antisense $5^{\prime}$-TCTTAGGTGTACTTTTGCCC-3'); Cpt1a (sense 5'-TCTTGCAGTCGACTCACCTT-3'; antisense 5'-TCCACAGGACACATAGTCAGG-3'), Tnfa (sense 5'-TGCCATCTCTGGGACAGG-3'; antisense 5'-TGGGAGTAGACAAGGTACAACC-3'); Ppara (sense 5'-GCTACCACTACGCAGTTCACG-3'; antisense 5'-GCTCCGATCACAACTTGTCGT-3'); Cd36 (sense 5'-CAAGCTCCTTGGCATGGTAGA; antisense 5'-TGGATTTGCAAGCACAATATGAA); G6pc (sense 5'-TTACCAGCCTCCTGTCGG; antisense 5'-GACACAACTGAAGCCGGTTAG); Ppargc1a (5'-TAAACTGAGCTACCCTTGG; antisense 5'-CTCGACACGGAGAGTTAAAGGAA); Foxo1 (5'-TGTTACTTAGCTCTCTCCCCTCG; antisense 5'-AGACGAGCAGTGGCTCAAT); Il6 (sense 5'-GTTCTCTGGGAAATCGTGGA; antisense 5'-TGTACTCCAAGCTATGG); chop (sense 5'-AAGGAGAAGGAGCAGGAGAAC; antisense 5'-GGTACACTTCCGGAGAGACAG). The relative quantification for a given gene was corrected to the cyclophilin mRNA values.

Human CHREBP expression was analyzed in human liver frozen tissues from 25 patients with histologically proven NASH. Liver mRNA was extracted in TRIzol solution, followed by an RNAeasy Kit (Qiagen). cDNAs were generated from $1 \mu \mathrm{g}$ RNA using Moloney murine leukemia virus (MMLV) reverse transcriptase (RT) (Invitrogen) and $\mathrm{pd}(\mathrm{N}) 6$ primers (Amersham Biosystems). Different amounts of calibrated mRNA (i.e., $1.25 \mathrm{pg}$ to $1.25 \times 10^{5} \mathrm{pg}$ ) (Applied Biosystems) were submitted to RT with the MMLV RT and $\mathrm{pd}(\mathrm{N}) 6$ primers and, subsequently, used in real-time PCR for standardization of HPRT transcripts. Briefly, the cDNA prepared from $200 \mathrm{ng}$ RNA was added to $50 \mu \mathrm{l}$ PCR buffer with 20 pmoles of each forward and reverse primers. The sequences of primers are as follows: hCHREBP, forward, 5'-TTTTGACCAGATGCGAGACA-3'; hCHREBP, reverse, 5'-TGGCGTAGGGAGTTCAGG-3'. qRT-PCR was performed using the SYBR green detection PCR Core Reagents Kit. Expression of target genes was achieved by the $\Delta \Delta \mathrm{C}_{\mathrm{t}}$ method (88).

Preparation of membrane and nuclear extracts. Liver nuclear and cytoplasmic extracts were prepared using the NE-PER Nuclear and Cytoplasmic Extraction Reagent Kit (Pierce Biotechnology) as previously described (17). Protein concentration was determined using the Bio-Rad Protein Assay.

Immunoblots analysis. Proteins $(40 \mu \mathrm{g})$ were subjected to SDS-PAGE analysis on $10 \%$ gels and transferred to nitrocellulose membranes. ChREBP protein was detected with a rabbit polyclonal antibody (Novus Biologicals), and SREBP-1c was detected with a mouse monoclonal antibody (SREBP-1 Ab-1; NeoMarkers, Interchim). Rabbit polyclonal ACACA, SCD1, NF-кB p65 and phospho-NF-кB p65 (Ser536), caspase-3, and PKCE antibodies were purchased from Cell Signaling. Insulin $\mathrm{R} \beta$ (C-19) was a rabbit polyclonal antibody raised against a peptide mapping at the $\mathrm{C}$ terminus of insulin R $\beta$ of human origin (IR-sc711; Santa Cruz Biotechnology Inc.). Rabbit polyclonal for total Akt, total GSK3 $\beta$, phospho-Akt (Ser473 and Thr308), phospho-GSK3 $\beta$ (Ser9), Akt substrates, and PCK1 antibodies were purchased from Cell Signaling. Monoclonal mouse $\beta$ actin (clone AC-74; Sigma-Aldrich) and lamin A/C (Cell Signaling) antibodies were used as loading controls. A polyclonal GFP antibody from Clontech was used. The FASN antibody was a gift from I. Dugail (Centre de Recherche des Cordeliers, Paris, France). The CRTC2 antibody was used as previously described (89). Autoradiograms of Western blots were scanned and quantified using an image processor program (Chemi Genius2 scan, SYNGENE).

Study population. Twenty-five patients with histologically proven NASH who were enrolled in the FLIRT trial, a randomized placebo-controlled fatty liver improvement with rosiglitazone therapy trial, were analyzed (48). The histologic diagnosis of NASH was defined as steatosis of more than $20 \%$ and the presence of either hepatocyte ballooning or intralobular hepa- tocyte necrosis. None of the patients had alcohol consumption of more than $20 \mathrm{~g}$ per day, hepatotoxic drugs, or treatment with insulin for diabetes or with ursodeoxycholic acid. The following diseases were excluded for each patient: cardiac insufficiency, neoplastic disease, current or past treatment with drugs that can induce steatohepatitis, uncompensated cirrhosis, organ transplantation, B and C viral hepatitis, autoimmune hepatitis, primary biliary cirrhosis, primary sclerosing cholangitis, biliary obstruction, hemochromatosis, and Wilson's disease.

Laboratory investigation. After a 12-hour overnight fast, venous blood samples were drawn to determine levels of AST, ALT, $\gamma$-glutamyltransferase, alkaline phosphatase, bilirubin, glucose, cholesterol, TG, and insulin. Results were obtained from a centralized laboratory. Insulin resistance was assessed by using the HOMA index as follows: fasting insulin $(\mathrm{mU} / \mathrm{l}) \times$ fasting plasma glucose $(\mathrm{mmol} / \mathrm{l}) / 22.5(90)$.

Histological investigation. Liver samples were paraffin embedded and stained with $\mathrm{H} \& \mathrm{E}$ safran and red sirius. Steatosis was semiquantitatively assessed as both the percentage of hepatocytes involved within a lobule $(0 \%-100 \%$, steatosis score) and by using a 4-grade classification modified from Kleiner et al. (91): 0 , absent; $1,<5 \% ; 2,5 \%-33 \% ; 3,33 \%-66 \% ; 4,>66 \%$. Staging and grading were performed according to Brunt et al. (92): F0, absence of any fibrosis; F1, isolated perisinusoidal or portal/periportal fibrosis; F2, perisinusoidal and portal/periportal fibrosis; F3, bridging fibrosis; F4, cirrhosis. The NAFLD activity score (NAS) was calculated according to Kleiner et al. (91). The score is defined as the sum of the scores for steatosis $(0,<5 \% ; 1,5 \%-33 \% ; 2,33 \%-66 \% ; 3,>66 \%)$, lobular inflammation ( 0 , none; $1,<2$ foci/ $\times 200$ magnification field; $2,2-4$ foci $\times 200$ magnification field; 3 , >4 foci/ $\times 200$ magnification field), and ballooning ( 0 , none; 1 , few; 2 , many). Thus, this score ranges from 0 to 8 . Fibrosis is not included in this score, as the separation of fibrosis from other features of activity is an accepted paradigm for staging and grading NASH (92). According to this classification, NASH is defined by a NAS of 5 or more.

Statistics. Statistical comparisons were made using ANOVA, $t$ test, or nonparametric Wilcoxon test in case of small groups. All data are presented as mean \pm SEM, with a statistically significant difference defined as a $P<0.05$.

Study approval. All procedures were carried out according to the French guidelines for the care and use of experimental animals. All animal studies were approved by the Direction Départementale des Services Vétérinaires de Paris and by the Mouse Facility Core laboratory (Institut Cochin, Inserm U1016, Université Paris Descartes, Paris, France). All patients gave written informed consent, and the study protocol was approved by the ethics committee of the Pitié-Salpêtrière Hospital.

\section{Acknowledgments}

The authors would like to thank Renaud Dentin and Anne-Françoise Burnol (Institut Cochin, Inserm U1016, Paris) for critical reading of the manuscript, Serge Luquet (EAC 7059 CNRS, Université Paris Diderot-Paris 7, Paris), Fabienne Rajas and Gilles Mithieux (Inserm U855/UCBL Faculté de Médecine Laennec, Lyon), Carina Prip-Buus and Abdelhak Mansouri (Institut Cochin, Inserm U1016, Paris) for helpful discussion, Howard C. Towle (University of Minnesota) for providing the ChREBPDP plasmid, Véronique Fauveau from the PlateForme of Chirurgie Experimentale (Institut Cochin, Inserm U1016, Paris) for performing adenoviral injections in mice, Bénédicte Noblet (Institut Cochin, Inserm U1016, Paris) for technical assistance, Maryline Favier from the Plate-forme de Morphologie/Histology (Institut Cochin, Inserm U1016, Paris) for performing liver sections and Oil Red O staining, Véronique Roques from the Lipidomic Facility (IFR-BMT, Toulouse) for performing GC analysis, and Anders Boyd for his assistance in statistics analysis (Inserm, UMRS 707, Paris). Mice used in this study were housed in an animal facility equipped 
with the help of the Région Ile de France. The work performed at the Cochin Institute was supported by grants from the Agence Nationale pour la Recherche (ANR) STRESSatose and CRISALIS, from the Association Française pour l'Etude du Foie (AFEF-2008), from the Fondation Française de la Recherche Médicale (Labélisation Equipe), and from the European Commission (FLORINASH, FP7 project); work performed at Centre de Recherche Saint-Antoine was supported by a grant from ANR-COD-2005, NASH-SIG.
Received for publication November 2, 2009, and accepted in revised form February 15, 2012.

Address correspondence to: Catherine Postic, Institut Cochin, Inserm U1016, Département d'Endocrinologie, Métabolisme et Cancer, 24 rue du faubourg Saint Jacques, Paris, 75014 France. Phone: 33.1.53.73.27.07; Fax: 33.1.44.41.24.21; E-mail: catherine. postic@inserm.fr.
1. Cheung O, Sanyal AJ. Recent advances in nonalcoholic fatty liver disease. Curr Opin Gastroenterol. 2010;26(3):202-208.

2. Dowman JK, Tomlinson JW, Newsome PN. Systematic review: the diagnosis and staging of non-alcoholic fatty liver disease and non-alcoholic steatohepatitis. Aliment Pharmacol Ther. 2011; 33(5):525-540.

3. Abdelmalek MF, Diehl AM. Nonalcoholic Fatty liver disease as a complication of insulin resistance. Med Clin North Am. 2007;91(6):1125-1149.

4. Charlton M. Nonalcoholic fatty liver disease: a review of current understanding and future impact. Clin Gastroenterol Hepatol. 2004;2(12):1048-1058.

5. Yamaguchi K, et al. Inhibiting triglyceride synthesis improves hepatic steatosis but exacerbates liver damage and fibrosis in obese mice with nonalcoholic steatohepatitis. Hepatology. 2007;45(6):1366-1374.

6. Listenberger LL, et al. Triglyceride accumulation protects against fatty acid-induced lipotoxicity. Proc Natl Acad Sci U S A. 2003;100(6):3077-3082.

7. Neuschwander-Tetri BA. Nontriglyceride hepatic lipotoxicity: the new paradigm for the pathogenesis of NASH. Curr Gastroenterol Rep. 2010;12(1):49-56.

8. Kumashiro N, et al. Cellular mechanism of insulin resistance in nonalcoholic fatty liver disease. Proc Natl Acad Sci U S A. 2011;108(39):16381-16385.

9. Monetti M, et al. Dissociation of hepatic steatosis and insulin resistance in mice overexpressing DGAT in the liver. Cell Metab. 2007;6(1):69-78.

10. Rinella ME, Green RM. The methionine-choline deficient dietary model of steatohepatitis does not exhibit insulin resistance. J Hepatol. 2004;40(1):47-51.

11. Buettner R, Ottinger I, Scholmerich J, Bollheimer LC. Preserved direct hepatic insulin action in rats with diet-induced hepatic steatosis. Am J Physiol Endocrinol Metab. 2004;286(5):E828-E833.

12. Stefan $\mathrm{N}$, et al. Identification and characterization of metabolically benign obesity in humans. Arch Intern Med. 2008;168(15):1609-1616.

13. Lodhi IJ, Wei X, Semenkovich CF. Lipoexpediency: de novo lipogenesis as a metabolic signal transmitter. Trends Endocrinol Metab. 2011;22(1):1-8.

14. Carobbio S, Rodriguez-Cuenca S, Vidal-Puig A. Origins of metabolic complications in obesity: ectopic fat accumulation. The importance of the qualitative aspect of lipotoxicity. Curr Opin Clin Nutr Metab Care. 2011;14(6):520-526.

15. Postic C, Girard J. Contribution of de novo fatty acid synthesis to hepatic steatosis and insulin resistance: lessons from genetically engineered mice. J Clin Invest. 2008;118(3):829-838.

16. Donnelly KL, Smith CI, Schwarzenberg SJ, Jessurun J, Boldt MD, Parks EJ. Sources of fatty acids stored in liver and secreted via lipoproteins in patients with nonalcoholic fatty liver disease. J Clin Invest. 2005;115(5):1343-1351.

17. Dentin R, et al. Hepatic glucokinase is required for the synergistic action of ChREBP and SREBP-1c on glycolytic and lipogenic gene expression. J Biol Chem. 2004;279(19):20314-20326.

18. Ishii S, Iizuka K, Miller BC, Uyeda K. Carbohydrate response element binding protein directly promotes lipogenic enzyme gene transcription. Proc Natl Acad Sci U S A. 2004;101(44):15597-15602.

19. Ma L, Tsatsos NG, Towle HC. Direct role of ChREBP.Mlx in regulating hepatic glucose-respon- sive genes. J Biol Chem. 2005;280(12):12019-12027.

20. Guinez C, et al. O-GlcNAcylation increases ChREBP protein content and transcriptional activity in the liver. Diabetes. 2011;60(5):1399-1413.

21. Bricambert J, Miranda J, Benhamed F, Girard J, Postic C, Dentin R. Salt-inducible kinase 2 links transcriptional coactivator $\mathrm{p} 300$ phosphorylation to the prevention of ChREBP-dependent hepatic steatosis in mice. J Clin Invest. 2010;120(12):4316-4331.

22. Kabashima T, Kawaguchi T, Wadzinski BE, Uyeda K. Xylulose 5-phosphate mediates glucose-induced lipogenesis by xylulose 5-phosphate-activated protein phosphatase in rat liver. Proc Natl Acad Sci US A. 2003;100(9):5107-5112.

23. Denechaud PD, et al. ChREBP, but not LXRs, is required for the induction of glucose-regulated genes in mouse liver. J Clin Invest. 2008;118(3):956-964.

24. Dentin R, et al. Glucose 6-phosphate, rather than xylulose 5-phosphate, is required for the activation of ChREBP in response to glucose in liver.J Hepatol. 2012;56(1):199-209.

25. Iizuka K, Bruick RK, Liang G, Horton JD, Uyeda K. Deficiency of carbohydrate response elementbinding protein (ChREBP) reduces lipogenesis as well as glycolysis. Proc Natl Acad Sci U S A. 2004; 101(19):7281-7286.

26. Iizuka K, Miller B, Uyeda K. Deficiency of carbohydrate-activated transcription factor ChREBP prevents obesity and improves plasma glucose control in leptin-deficient (ob/ob) mice. Am J Physiol Endocrinol Metab. 2006;291(2):E358-E364.

27. Dentin R, et al. Liver-specific inhibition of ChREBP improves hepatic steatosis and insulin resistance in ob/ob mice. Diabetes. 2006;55(8):2159-2170.

28. Foretz M, Guichard C, Ferré P, Foufelle F. Sterol regulatoy element binding protein-1c is a major mediator of insulin action on the hepatic expression of glucokinase and lipogenesis-related genes. Proc Natl Acad Sci U S A. 1999;96(22):12737-12742.

29. Virtue S, Vidal-Puig A. It's not how fat you are, it's what you do with it that counts. PLoS Biol. 2008; 6(9):e237.

30. Holland WL, Knotts TA, Chavez JA, Wang LP, Hoehn KL, Summers SA. Lipid mediators of insulin resistance. Nutr Rev. 2007;65(6 pt 2):S39-S46.

31. Samuel VT, et al. Mechanism of hepatic insulin resistance in non-alcoholic fatty liver disease. J Biol Chem. 2004;279(31):32345-32353.

32. Samuel VT, et al. Inhibition of protein kinase Cepsilon prevents hepatic insulin resistance in nonalcoholic fatty liver disease. J Clin Invest. 2007; 117(3):739-745.

33. Badman MK, Pissios P, Kennedy AR, Koukos G, Flier JS, Maratos-Flier E. Hepatic fibroblast growth factor 21 is regulated by PPARalpha and is a key mediator of hepatic lipid metabolism in ketotic states. Cell Metab. 2007;5(6):426-437.

34. Iizuka K, Takeda J, Horikawa Y. Glucose induces FGF21 mRNA expression through ChREBP activation in rat hepatocytes. FEBS Lett. 2009; 583(17):2882-2886

35. Flowers MT, Ntambi JM. Role of stearoyl-coenzyme A desaturase in regulating lipid metabolism. Curr Opin Lipidol. 2008;19(3):248-256

36. Paton CM, Ntambi JM. Loss of stearoyl-CoA desaturase activity leads to free cholesterol synthesis through increased Xbp-1 splicing. Am J Physiol
Endocrinol Metab. 2010;299(6):E1066-E1075.

37. Sampath H, Ntambi JM. The role of stearoyl-CoA desaturase in obesity, insulin resistance, and inflammation. Ann N Y Acad Sci. 2011;1243(1):47-53.

38. Miyazaki M, et al. Hepatic stearoyl-CoA desaturase-1 deficiency protects mice from carbohydrate-induced adiposity and hepatic steatosis. Cell Metab. 2007; 6(6):484-496

39. Ariyama H, Kono N, Matsuda S, Inoue T, Arai H. Decrease in membrane phospholipid unsaturation induces unfolded protein response. J Biol Chem. 2010; 285(29):22027-22035.

40. Thorn K, Hovsepyan M, Bergsten P. Reduced levels of SCD1 accentuate palmitate-induced stress in insulin-producing beta-cells. Lipids Health Dis. 2010; 9:108.

41. Ron D, Walter P. Signal integration in the endoplasmic reticulum unfolded protein response. Nat Rev Mol Cell Biol. 2007;8(7):519-529.

42. Flamment M, Kammoun HL, Hainault I, Ferre P, Foufelle F. Endoplasmic reticulum stress: a new actor in the development of hepatic steatosis. Curr Opin Lipidol. 2010;21(3):239-246.

43. Louvet A, et al. Cannabinoid CB2 receptors protect against alcoholic liver disease by regulating Kupffer cell polarization in mice. Hepatology. 2011; 54(4):1217-1226.

44. Puigserver $P$, etal. Insulin-regulated hepatic gluconeogenesis through FOXO1-PGC-1alpha interaction. Nature. 2003;423(6939):550-555.

45. Dentin R, et al. Insulin modulates gluconeogenesis by inhibition of the coactivator TORC2. Nature. 2007; 449(7160):366-369.

46. Zhang EE, et al. Cryptochrome mediates circadian regulation of CAMP signaling and hepatic gluconeogenesis. Nat Med. 2010;16(10):1152-1156.

47. Kharitonenkov A, Shanafelt AB. Fibroblast growth factor-21 as a therapeutic agent for metabolic diseases. BioDrugs. 2008;22(1):37-44.

48. Ratziu V, et al. Rosiglitazone for nonalcoholic steatohepatitis: one-year results of the randomized placebo-controlled Fatty Liver Improvement with Rosiglitazone Therapy (FLIRT) Trial. Gastroenterology. 2008;135(1):100-110.

49. Marchesini G, et al. Nonalcoholic fatty liver disease: a feature of the metabolic syndrome. Diabetes. 2001;50(8):1844-1850.

50. Anstee QM, Goldin RD. Mouse models in nonalcoholic fatty liver disease and steatohepatitis research. Int J Exp Pathol. 2006;87(1):1-16.

51. Tsatsos NG, Towle HC. Glucose activation of ChREBP in hepatocytes occurs via a two-step mechanism. Biochem Biophys Res Commun. 2006; 340(2):449-456

52. Guha P, Aneja KK, Shilpi RY, Haldar D. Transcriptional regulation of mitochondrial glycerophosphate acyltransferase is mediated by distal promoter via ChREBP and SREBP-1. Arch Biochem Biophys. 2009;490(2):85-95.

53. Jeong YS, et al. Integrated expression profiling and genome-wide analysis of ChREBP targets reveals the dual role for ChREBP in glucose-regulated gene expression. PLoS One. 2011;6(7):e22544.

54. Neuschwander-Tetri BA. Hepatic lipotoxicity and the pathogenesis of nonalcoholic steatohepatitis: the central role of nontriglyceride fatty acid metabolites. Hepatology. 2010;52(2):774-788. 
55. Farese RV Jr, Walther TC. Lipid droplets finally get a little R-E-S-P-E-C-T. Cell. 2009;139(5):855-860.

56. Koliwad SK, et al. DGAT1-dependent triacylglycerol storage by macrophages protects mice from diet-induced insulin resistance and inflammation. J Clin Invest. 2010;120(3):756-767.

57. Son $\mathrm{NH}$, et al. PPARgamma-induced cardiolipotoxicity in mice is ameliorated by PPARalpha deficiency despite increases in fatty acid oxidation. J Clin Invest. 2010;120(10):3443-3454.

58. Timmers S, et al. Prevention of high-fat diet-induced muscular lipid accumulation in rats by alpha lipoic acid is not mediated by AMPK activation. J Lipid Res. 2010;51(2):352-359.

59. Guillou H, Zadravec D, Martin PG, Jacobsson A. The key roles of elongases and desaturases in mammalian fatty acid metabolism: Insights from transgenic mice. Prog Lipid Res. 2010;49(2):186-199.

60. Moon YA, Hammer RE, Horton JD. Deletion of ELOVL5 leads to fatty liver through activation of SREBP-1c in mice. J Lipid Res. 2009;50(3):412-423.

61. Akazawa $Y$, et al. Palmitoleate attenuates palmitateinduced Bim and PUMA up-regulation and hepatocyte lipoapoptosis. J Hepatol. 2010;52(4):586-593.

62. Cazanave SC, Elmi NA, Akazawa Y, Bronk SF, Mott JL, Gores GJ. CHOP and AP-1 cooperatively mediate PUMA expression during lipoapoptosis. Am J Physiol Gastrointest Liver Physiol. 2010;299(1):G236-G243.

63. Miyazaki M, et al. Stearoyl-CoA desaturase 1 gene expression is necessary for fructose-mediated induction of lipogenic gene expression by sterol regulatory element-binding protein-1c-dependent and -independent mechanisms. J Biol Chem. 2004 279(24):25164-25171.

64. Flowers JB, et al. Loss of stearoyl-CoA desaturase-1 improves insulin sensitivity in lean mice but worsens diabetes in leptin-deficient obese mice. Diabetes. 2007; 56(5):1228-1239.

65. Li ZZ, Berk M, McIntyre TM, Feldstein AE. Hepatic lipid partitioning and liver damage in nonalcoholic fatty liver disease: role of stearoyl-CoA desaturase. J Biol Chem. 2009;284(9):5637-5644.

66. Chen C, et al. Metabolomics reveals that hepatic stearoyl-CoA desaturase 1 downregulation exacerbates inflammation and acute colitis. Cell Metab. 2008; $7(2): 135-147$.

67. Tardif $\mathrm{N}$, et al. Oleate-enriched diet improves insu- lin sensitivity and restores muscle protein synthesis in old rats. Clin Nutr. 2011;30(6):799-806.

68. Minehira K, et al. Blocking VLDL secretion causes hepatic steatosis but does not affect peripheral lipid stores or insulin sensitivity in mice. J Lipid Res. 2008; 49(9):2038-2044.

69. Chen KS, Heydrick SJ, Brown ML, Friel JC, Ruderman NB. Insulin increases a biochemically distinct pool of diacylglycerol in the rat soleus muscle. Am J Physiol. 1994;266(3 pt 1):E479-E485.

70. Nagle CA, Klett EL, Coleman RA. Hepatic triacylglycerol accumulation and insulin resistance. J Lipid Res. 2009;50 suppl:S74-S79.

71. Wu C, et al. Enhancing hepatic glycolysis reduces obesity: differential effects on lipogenesis depend on site of glycolytic modulation. Cell Metab. 2005; 2(2):131-140

72. de Luis O, Valero MC, Jurado LA. WBSCR14, a putative transcription factor gene deleted in Williams-Beuren syndrome: complete characterisation of the human gene and the mouse ortholog. Eur J Hum Genet. 2000;8(3):215-222.

73. Noriega LG, et al. CREB and ChREBP oppositely regulate SIRT1 expression in response to energy availability. EMBO Rep. 2011;12(10):1069-1076.

74. Rodgers JT, Puigserver P. Fasting-dependent glucose and lipid metabolic response through hepatic sirtuin 1. Proc Natl Acad Sci U S A. 2007; 104(31):12861-12866.

75. Liu Y, et al. A fasting inducible switch modulates gluconeogenesis via activator/coactivator exchange. Nature. 2008;456(7219):269-273.

76. Canto C, Auwerx J. PGC-1alpha, SIRT1 and AMPK, an energy sensing network that controls energy expenditure. Curr Opin Lipidol. 2009;20(2):98-105.

77. Rodgers JT, Lerin C, Haas W, Gygi SP, Spiegelman BM, Puigserver P. Nutrient control of glucose homeostasis through a complex of PGC-1alpha and SIRT1. Nature. 2005;434(7029):113-118.

78. Kohjima M, et al. Re-evaluation of fatty acid metabolism-related gene expression in nonalcoholic fatty liver disease. Int J Mol Med. 2007;20(3):351-358.

79. Hurtado Del Pozo C, et al. ChREBP expression in the liver, adipose tissue and differentiated preadipocytes in human obesity. Biochim Biophys Acta. 2011;1811(12):1194-1200.

80. Kantartzis K, et al. Dissociation between fatty liver and insulin resistance in humans carrying a variant of the patatin-like phospholipase 3 gene. Diabetes. 2009;58(11):2616-2623.

81. Semple RK, et al. Postreceptor insulin resistance contributes to human dyslipidemia and hepatic steatosis. J Clin Invest. 2009;119(2):315-322.

82. Dentin R, et al. Liver-specific inhibition of ChREBP improves hepatic steatosis and insulin resistance in ob/ob mice. Diabetes. 2006;55(8):2159-2170.

83. Green H, Kehinde O. An established preadipose cell line and its differentiation in culture. II. Factors affecting the adipose conversion. Cell. 1975; 5(1):19-27.

84. Carre N, Cauzac M, Girard J, Burnol AF. Dual effect of the adapter growth factor receptor-bound protein 14 (grb14) on insulin action in primary hepatocytes. Endocrinology. 2008;149(6):3109-3117.

85. Barrans A, et al. Hepatic lipase induces the formation of pre-beta 1 high density lipoprotein (HDL) from triacylglycerol-rich HDL2. A study comparing liver perfusion to in vitro incubation with lipases. J Biol Chem. 1994;269(15):11572-11577.

86. Vieu C, et al. Coupled assay of sphingomyelin and ceramide molecular species by gas liquid chromatography. J Lipid Res. 2002;43(3):510-522.

87. El Hadri K, et al. In vitro suppression of the lipogenic pathway by the nonnucleoside reverse transcriptase inhibitor efavirenz in 3T3 and human preadipocytes or adipocytes. J Biol Chem. 2004; 279(15):15130-15141.

88. Livak KJ, Schmittgen TD. Analysis of relative gene expression data using real-time quantitative PCR and the 2(-Delta Delta C(T)) Method. Methods. 2001; 25(4):402-408.

89. Dentin R, Hedrick S, Xie J, Yates J 3rd, Montminy $M$. Hepatic glucose sensing via the CREB coactivator CRTC2. Science. 2008;319(5868):1402-1405.

90. Wallace TM, Matthews DR. The assessment of insulin resistance in man. Diabet Med. 2002;19(7):527-534.

91. Kleiner DE, et al. Design and validation of a histological scoring system for nonalcoholic fatty liver disease. Hepatology. 2005;41(6):1313-1321.

92. Brunt EM, Janney CG, Di Bisceglie AM, Neuschwander-Tetri BA, Bacon BR. Nonalcoholic steatohepatitis: a proposal for grading and staging the histological lesions. Am J Gastroenterol. 1999; 94(9):2467-2474 